\title{
Dispersion estimates for one-dimensional discrete Schrödinger and wave equations
}

\author{
Iryna Egorova, ${ }^{1}$ Elena Kopylova, ${ }^{1}$ and Gerald Teschl ${ }^{1}$
}

\begin{abstract}
We derive dispersion estimates for solutions of the one-dimensional discrete perturbed Schrödinger and wave equations. In particular, we improve upon previous works and weaken the conditions on the potentials. To this end we also provide new results concerning scattering for one-dimensional discrete perturbed Schrödinger operators which are of independent interest. Most notably we show that the reflection and transmission coefficients belong to the Wiener algebra.
\end{abstract}

Mathematics Subject Classification (2010). Primary: 35Q41, 81Q15; Secondary: 39A12, 39A70.

Keywords. Discrete Schrödinger equation, discrete wave equation, dispersive decay, scattering theory.

\section{Contents}

1 Introduction . . . . . . . . . . . . . . . . . . . . . . . 664

2 Free discrete Schrödinger equation . . . . . . . . . . . . . 666

3 Jost solutions and the resolvent . . . . . . . . . . . . . . . . . . 671

4 Properties of the scattering matrix . . . . . . . . . . . . . . . . 674

5 Dispersive decay in the resonant case . . . . . . . . . . . . . 677

6 Dispersive decay in the non-resonant case . . . . . . . . . . . . . 681

7 Wave equation . . . . . . . . . . . . . . . . 687

References . . . . . . . . . . . . . . . . . . . . . . . 694

\footnotetext{
${ }^{1}$ Research supported by the Austrian Science Fund (FWF) under Grant No. Y330, M1329 and RFBR grants.
} 


\section{Introduction}

We are concerned with the one-dimensional discrete Schrödinger equation

$$
\mathrm{i} \dot{u}(t)=H u(t):=\left(-\Delta_{L}+q\right) u(t), \quad t \in \mathbb{R},
$$

and the corresponding discrete wave (resp. Klein-Gordon) equation

$$
\ddot{u}(t)=\left(\Delta_{L}-\mu^{2}-q\right) u(t), \quad t \in \mathbb{R}, \mu \geq 0 .
$$

with real potential $q$. Here $\Delta_{L}$ is the discrete Laplacian given by

$$
\left(\Delta_{L} u\right)_{n}=u_{n+1}-2 u_{n}+u_{n-1}, \quad n \in \mathbb{Z} .
$$

To formulate our results we introduce the weighted spaces $\ell_{\sigma}^{p}=\ell_{\sigma}^{p}(\mathbb{Z}), \sigma \in \mathbb{R}$, associated with the norm

$$
\|u\|_{\ell_{\sigma}^{p}}= \begin{cases}\left(\sum_{n \in \mathbb{Z}}(1+|n|)^{p \sigma}|u(n)|^{p}\right)^{1 / p}, & p \in[1, \infty), \\ \sup _{n \in \mathbb{Z}}(1+|n|)^{\sigma}|u(n)|, & p=\infty .\end{cases}
$$

Of course, the case $\sigma=0$ corresponds to the usual $\ell_{0}^{p}=\ell^{p}$ spaces without weight.

As our first main result we will prove the following $\ell^{1} \rightarrow \ell^{\infty}$ decay

$$
\left\|\mathrm{e}^{-\mathrm{i} t H} P_{c}\right\|_{\ell^{1} \rightarrow \ell \infty}=\mathcal{O}\left(t^{-1 / 3}\right), \quad t \rightarrow \infty,
$$

under the assumption $q \in \ell_{1}^{1}$. Here $P_{c}$ is the orthogonal projection in $\ell^{2}$ onto the continuous spectrum of $H$. In this respect we recall that under the condition $q \in \ell_{1}^{1}$ it is well-known [20] that the spectrum of $H$ consists of a purely absolutely continuous part covering $[0,4]$ plus a finite number of eigenvalues located in $\mathbb{R} \backslash[0,4]$. In addition, there could be resonances at the edges of the continuous spectrum.

The dispersive decay (1.3) has been established by Pelinovsky and Stefanov [16] under the assumption that there are no resonances and under the more restrictive condition $\left|q_{n}\right| \leq C(1+|n|)^{-\beta}$ with $\beta>5$. Cuccagna and Tarulli [1] establish (1.3) under the assumption $q \in \ell_{1}^{1}$ if there are no resonances and under the assumption $q \in \ell_{2}^{1}$ if there are resonances (which compares to the continuous case established in [8]). Our main contribution here is to show that this extra decay condition in the case of resonances is not necessary. Our novel proof is based on a simple but useful generalization of the van der Corput lemma (Lemma 5.1) together with the novel fact that the scattering data associated with $H$ are in the 
Wiener algebra (Theorem 4.1). The latter result being of independent interest in scattering theory.

Moreover, (1.3) has some immediate consequences (under the same assumption $\left.q \in \ell_{1}^{1}\right)$. First of all unitarity of $\exp (-\mathrm{i} t H): \ell^{2} \rightarrow \ell^{2}$ implies

$$
\left\|\mathrm{e}^{-\mathrm{i} t H} P_{c}(H)\right\|_{\ell^{2} \rightarrow \ell^{2}} \leq 1
$$

and interpolating between this and our $\ell^{1} \rightarrow \ell^{\infty}$ estimate the Riesz-Thorin theorem gives

$$
\left\|\mathrm{e}^{-\mathrm{i} t H} P_{c}(H)\right\|_{\ell p^{\prime} \rightarrow \ell p}=\mathcal{O}\left(t^{-1 / 3\left(1 / p^{\prime}-1 / p\right)}\right)
$$

for any $p^{\prime} \in[1,2]$ with $\frac{1}{p}+\frac{1}{p^{\prime}}=1$. Moreover, we also can deduce some corresponding Strichartz estimates from Theorem 1.2 of [11]. To this end we introduce the following space-time norms

$$
\|F\|_{L^{q}, \ell^{p}}=\left(\int_{\mathbb{R}}\|F(t)\|_{\ell^{p}}^{q} d t\right)^{1 / q} .
$$

Then

$$
\begin{aligned}
\left\|\mathrm{e}^{-\mathrm{i} t H} P_{c}(H) f\right\|_{L^{q}, \ell^{p}} & \leq C\|f\|_{\ell^{2}}, \\
\left\|\int_{\mathbb{R}} \mathrm{e}^{-\mathrm{i} s H} P_{c}(H) F(s) d s\right\|_{\ell^{2}} & \leq C\|F\|_{L^{q^{\prime}, \ell^{p^{\prime}}},}, \\
\left\|\int_{s<t} \mathrm{e}^{-\mathrm{i}(t-s) H} P_{c}(H) F(s) d s\right\|_{L^{q}, \ell^{p}} & \leq C\|F\|_{L^{q^{\prime}}, \ell^{p^{\prime}}},
\end{aligned}
$$

where $p, q \geq 2$,

$$
\frac{1}{q}+\frac{1}{3 p} \leq \frac{1}{6}
$$

and a prime denotes the corresponding dual index. Furthermore, (1.3) also implies

$$
\left\|\mathrm{e}^{-\mathrm{i} t H} P_{c}(H)\right\|_{\ell_{\sigma}^{2} \rightarrow \ell_{-}^{2}}=\mathcal{O}\left(t^{-1 / 3}\right), \quad t \rightarrow \infty, \sigma>1 / 2 .
$$

However, we will in fact establish the stronger result

$$
\left\|\mathrm{e}^{-\mathrm{i} t H} P_{c}\right\|_{\ell_{\sigma}^{2} \rightarrow \ell_{-\sigma}^{2}}=\mathcal{O}\left(t^{-1 / 2}\right), \quad t \rightarrow \infty, \sigma>1 / 2,
$$

which has not been obtained previously.

For the remaining results we restrict ourselves to the case when the edges of the spectrum $\omega=0,4$ are no resonances for the operator $H$. Then for $q \in \ell_{2}^{1}$ we show that

$$
\left\|\mathrm{e}^{-\mathrm{i} t H} P_{c}\right\|_{\ell_{1}^{1} \rightarrow \ell_{-1}^{\infty}}=\mathcal{O}\left(t^{-4 / 3}\right), \quad t \rightarrow \infty .
$$

Such asymptotics with decay rate $t^{-3 / 2}$ were first established for continuous Schrödinger equations by Schlag in [17] in the case when the potential has a finite fourth 
moment and later refined by Goldberg [7] to the case of a finite third moment. For the discrete Schrödinger equations again asymptotics of the type (1.9) appear to be new.

Moreover, for $q \in \ell_{2}^{1}$ in the non-resonant case we prove

$$
\left\|\mathrm{e}^{-\mathrm{i} t H} P_{c}\right\|_{\ell_{\sigma}^{2} \rightarrow \ell_{-\sigma}^{2}}=\mathcal{O}\left(t^{-3 / 2}\right), \quad t \rightarrow \infty, \sigma>3 / 2
$$

Such a dispersive decay estimate was obtained for the first time in [13] for discrete Schrödinger and Klein-Gordon equations with compactly supported potentials. The result has been generalized in [16] to discrete Schrödinger equation with noncompactly supported potentials under the decay condition $\left|q_{n}\right| \leq C(1+|n|)^{-\beta}$ with $\beta>5$ and for $\sigma>5 / 2$.

Here we improve this result by both reducing the decay rate and the value of $\sigma$. Again, this reduction relies on our new approach based on properties of the Jost functions and the scattering matrix.

Finally, we obtain similar asymptotics for the wave (resp. Klein-Gordon) equation (1.2) (except for the asymptotics in the resonant case when $\mu=0$ ).

In addition, we mention that asymptotics of the type (1.3)-(1.10) play an important role in proving asymptotic stability of solitons in the associated discrete nonlinear equations $[10,14,15,18]$. Analogous results for the continuous one dimensional Schrödinger and Klein-Gordon equations will be given in [5].

\section{Free discrete Schrödinger equation}

As a warm-up we will first consider the free equation (1.1) with $q=0$ and denote $H_{0}=-\Delta_{L}$. It is well-known ([20, Sect. 1.3]) that $H_{0}$ is self-adjoint and the discrete Fourier transform

$$
\hat{u}(\theta)=\sum_{n \in \mathbb{Z}} u_{n} \mathrm{e}^{\mathrm{i} \theta n}, \quad \theta \in \mathbb{T}:=\mathbb{R} / 2 \pi \mathbb{Z} .
$$

maps $H_{0}$ to the operator of multiplication by $\phi(\theta)=2-2 \cos \theta$ :

$$
\widehat{H_{0} u}(\theta)=\phi(\theta) \widehat{u}(\theta)
$$

In particular, the spectrum $\operatorname{Spec}\left(H_{0}\right)=[0,4]$ is purely absolutely continuous. 
Adopting the notation $[K]_{n, k}$ for the kernel of an operator $K$, that is,

$$
(K u)_{n}=\sum_{k \in \mathbb{Z}}[K]_{n, k} u_{k}, \quad n \in \mathbb{Z},
$$

the kernel of the resolvent $\mathcal{R}_{0}(\omega)=\left(H_{0}-\omega\right)^{-1}$ is given by (cf. [13])

$$
\left[\mathcal{R}_{0}(\omega)\right]_{n, k}=\frac{1}{2 \pi} \int_{\mathbb{T}} \frac{\mathrm{e}^{-\mathrm{i} \theta(n-k)}}{\phi(\theta)-\omega} d \theta=\frac{\mathrm{e}^{-\mathrm{i} \theta(\omega)|n-k|}}{2 \mathrm{i} \sin \theta(\omega)}, \quad \omega \in \Xi:=\mathbb{C} \backslash[0,4],
$$

$n, k \in \mathbb{Z}$. Here $\theta(\omega)$ is the unique solution of the equation

$$
2-2 \cos \theta=\omega, \quad \theta \in \Sigma:=\{-\pi \leq \operatorname{Re} \theta \leq \pi, \operatorname{Im} \theta<0\} .
$$

Observe that $\theta \mapsto \omega=2-2 \cos \theta$ is a biholomorphic map from $\Sigma \rightarrow \Xi$ with identified points $\theta=-\pi-\mathrm{i} a$ and $\theta=\pi-\mathrm{i} a, a \geq 0$. Then the map $z=\mathrm{e}^{-\mathrm{i} \theta}$ is one-to-one from $\Sigma$ to the interior of the unit circle $|z|<1$. Note that the parameter $z$ is the standard spectral parameter for the Jacobi difference equation $a_{n-1} u_{n-1}+b_{n} u_{n}+a_{n} u_{n+1}=\left(z+z^{-1}\right) u_{n}$, where $a_{n} \rightarrow 1$ and $b_{n} \rightarrow 0$ as $n \rightarrow \pm \infty$. The scattering theory of this equation can be found in [20].

The kernel of the free propagator can be easily computed using the spectral theorem

$$
\begin{aligned}
{\left[\mathrm{e}^{-\mathrm{i} t H_{0}}\right]_{n, k} } & =\frac{1}{2 \pi \mathrm{i}} \int_{[0,4]} \mathrm{e}^{-\mathrm{i} t \omega}\left[\mathcal{R}_{0}(\omega+\mathrm{i} 0)-\mathcal{R}_{0}(\omega-\mathrm{i} 0)\right]_{n, k} d \omega \\
& =-\frac{1}{4 \pi} \int_{[0,4]} \mathrm{e}^{-\mathrm{i} t \omega}\left(\frac{\mathrm{e}^{-\mathrm{i} \theta+(\omega)|n-k|}}{\sin \theta_{+}(\omega)}-\frac{\mathrm{e}^{-\mathrm{i} \theta-(\omega)|n-k|}}{\sin \theta_{-}(\omega)}\right) d \omega \\
& =\frac{1}{2 \pi} \int_{-\pi}^{\pi} \mathrm{e}^{-\mathrm{i} t(2-2 \cos \theta)} \mathrm{e}^{-\mathrm{i} \theta|n-k|} d \theta
\end{aligned}
$$

where

$$
\theta_{+}(\omega)=\theta(\omega+\mathrm{i} 0) \in[-\pi, 0], \quad \theta_{-}(\omega)=\theta(\omega-\mathrm{i} 0) \in[0, \pi], \quad \omega \in[0,4] .
$$

The last integral in (2.3) is Bessel's integral implying

$$
\left[\mathrm{e}^{-\mathrm{i} t H_{0}}\right]_{n, k}=\mathrm{e}^{\mathrm{i}\left(-2 t+\frac{\pi}{2}|n-k|\right)} J_{|n-k|}(2 t),
$$

where $J_{v}(z)$ denotes the Bessel function of order $v$, [21].

For the free discrete Schrödinger equation the $\ell^{1} \rightarrow \ell^{\infty}$ decay and the $\ell_{\sigma}^{2} \rightarrow \ell_{-\sigma}^{2}$ decay holds only with the rates $t^{-1 / 3}$ and $t^{-1 / 2}$, respectively (the latter one being the same as in the continuous case). This is caused by the presence of resonances at the edge points $\omega=0$ and $\omega=4$. 
Proposition 2.1. The following asymptotics hold

$$
\begin{aligned}
\left\|\mathrm{e}^{-\mathrm{i} t H_{0}}\right\|_{\ell^{1} \rightarrow \ell^{\infty}} & =\mathcal{O}\left(t^{-1 / 3}\right), \quad t \rightarrow \infty, \\
\left\|\mathrm{e}^{-\mathrm{i} t H_{0}}\right\|_{\ell_{\sigma}^{2} \rightarrow \ell_{-\sigma}^{2}} & =\mathcal{O}\left(t^{-1 / 2}\right), \quad t \rightarrow \infty, \sigma>1 / 2 .
\end{aligned}
$$

Proof. STEP I. To establish (2.6) consider $t \geq 1$ and set

$$
v:=|n-k| / t \geq 0 .
$$

We start from

$$
\left[\mathrm{e}^{-\mathrm{i} t H_{0}}\right]_{n, k}=\frac{1}{2 \pi} \int_{-\pi}^{\pi} \mathrm{e}^{-\mathrm{i} t(2-2 \cos \theta+v \theta)} d \theta
$$

which is an oscillatory integral with the phase function

$$
\phi_{v}(\theta)=2-2 \cos \theta+v \theta .
$$

The stationary points are the solution of the equation $\phi_{v}^{\prime}(\theta)=2 \sin \theta+v=0$. If $v>2$ the phase function has no stationary points. For any $v<2$ the phase function has two stationary points $\theta_{1,2}$, which are non-degenerate, i.e. $\phi_{v}^{\prime \prime}\left(\theta_{1,2}\right) \neq 0$. In the case $v=2$ the phase function has a unique degenerate stationary point $\theta=-\pi / 2$ satisfying

$$
\phi_{2}^{\prime \prime}(-\pi / 2)=0, \quad \phi_{2}^{\prime \prime \prime}(-\pi / 2)=2 \neq 0 .
$$

Then, since $\phi_{v}^{\prime \prime}(\theta)=2 \cos (\theta)$ and $\phi_{v}^{\prime \prime \prime}(\theta)=-2 \sin (\theta)$, we can split our domain of integration into four intervals where either $\left|\phi_{v}^{\prime \prime}(\theta)\right| \geq \sqrt{2}$ or $\left|\phi_{v}^{\prime \prime \prime}(\theta)\right| \geq \sqrt{2}$. Applying the van der Corput lemma [19, page 334] on each interval gives (2.6).

$\mathrm{S}_{\text {TEP II }}$. To establish (2.7) we represent $\mathrm{e}^{-\mathrm{i} t H_{0}}$ as the sum

$$
\mathrm{e}^{-\mathrm{i} t H_{0}}=\frac{1}{2 \pi}(K(t)+\widetilde{K}(t))
$$

where

$$
[K(t)]_{n, k}=\int_{\left|\theta+\frac{\pi}{2}\right| \leq \frac{\pi}{6}} \mathrm{e}^{-\mathrm{i} t \phi_{v}(\theta)} d \theta, \quad[\tilde{K}(t)]_{n, k}=\int_{\left|\theta+\frac{\pi}{2}\right| \geq \frac{\pi}{6}} \mathrm{e}^{-\mathrm{i} t \phi_{v}(\theta)} d \theta .
$$

By the stationary phase method we infer

$$
\sup _{n, k \in \mathbb{Z}}\left|[\tilde{K}(t)]_{n, k}\right| \leq C t^{-1 / 2}, \quad t \geq 1,
$$

implying (2.7) for $\tilde{K}(t)$. The required estimate for $K(t)$ will follow from the next lemma. 
Lemma 2.2. For any $\sigma>1 / 2$ the following estimate holds:

$$
\sum_{n, k \in \mathbb{Z}}[K(t)]_{n, k}^{2} \frac{1}{(1+|n|)^{2 \sigma}(1+|k|)^{2 \sigma}} \leq C t^{-1} .
$$

Proof. For any fixed $\sigma>1 / 2$, there exist an integer $N>0$ such that

$$
\sigma>1 / 2+(1 / 2)^{N} .
$$

Denote $t_{j}=t^{-\left(\frac{1}{2}\right)^{j}}, 1 \leq j \leq N, t_{0}=0, t_{N+1}=\pi / 6$, and represent $K(t)$ as the sum

$$
K(t)=\sum_{j=0}^{N} K_{j}(t)
$$

where $K_{j}(t), 0 \leq j \leq N$, is the integral over $t_{j} \leq\left|\theta+\frac{\pi}{2}\right| \leq t_{j+1}$. We will establish a bound of type (2.11) for each summand. For $K_{0}(t)$ the bound evidently holds. Furthermore, by the van der Corput lemma

$$
\sup _{n, k \in \mathbb{Z}}\left|\int_{t_{j} \leq\left|\theta+\frac{\pi}{2}\right| \leq a} \mathrm{e}^{-\mathrm{i} t \phi_{v}(\theta)} d \theta\right| \leq C t^{-1 / 2}\left(\min _{t_{j} \leq\left|\theta+\frac{\pi}{2}\right| \leq \frac{\pi}{6}}\left|\phi_{v}^{\prime \prime}(\theta)\right|\right)^{-1 / 2} \leq C\left(t t_{j}\right)^{-1 / 2}
$$

for any $a \in\left[t_{j}, \pi / 6\right]$ implying

$$
\sup _{n, k \in \mathbb{Z}}\left|\left[K_{j}(t)\right]_{n, k}\right| \leq C t^{-1 / 2} t_{j}^{-1 / 2}, \quad j=1, \ldots, N .
$$

To get the estimate (2.11) for each $K_{j}(t), 1 \leq j \leq N$, we choose $\varepsilon=2^{-N}$, so that $t^{\varepsilon}=t_{N}^{-1}$, and consider two different cases: $|2-v| \in\left[0, t_{j} t^{\varepsilon}\right]$ and $|2-v| \in\left[t_{j} t^{\varepsilon}, 2\right]$ separately.

In the first case we take

$$
T_{j}=\left\{(n, k) \in \mathbb{Z}^{2}:|2 t-| n-k|| \leq t_{j} t^{1+\varepsilon}\right\}
$$

as the domain of summation. Since this domain is symmetric with respect to the map $(n, k) \mapsto(-n,-k)$, we can make the change of variables $p=n-k, q=n+k$ and estimate

$$
b_{j}(t):=\sum_{(n, k) \in T_{j}} \frac{1}{(1+|n|)^{2 \sigma}(1+|k|)^{2 \sigma}}
$$

as

$$
b_{j}(t) \leq \sum_{q \in \mathbb{Z}} \sum_{p=\left\lceil 2 t-t_{j} t^{1+\varepsilon}\right\rceil}^{\left\lfloor 2 t+t_{j} t^{1+\varepsilon}\right\rfloor} \frac{2}{\left(1+\frac{1}{2}|p+q|\right)^{2 \sigma}\left(1+\frac{1}{2}|p-q|\right)^{2 \sigma}},
$$


where $\lfloor\cdot],\lceil\cdot\rceil$ denote the usual floor and ceiling functions. The sum with respect to $p$ is finite with the number of summands less then $2\left\lfloor t_{j} t^{1+\varepsilon}\right\rfloor+2$. Since $t_{j} t^{1+\varepsilon} \leq t$ for $j=1, \ldots, N$ we have $p \geq t$ in the domain of summation. Consequently $p+q \geq t$ for $q \geq 0$ and $p-q \geq t$ for $q<0$. Using these estimates and interchanging the order of summation we get

$$
b_{j}(t) \leq C \frac{\left\lfloor t_{j} t^{1+\varepsilon}\right\rfloor}{t^{2 \sigma}} \leq C t_{j} t^{1+\varepsilon-2 \sigma} .
$$

Thus, by (2.14), (2.15), and (2.12)

$$
\sum_{n, k \in T_{j}} \frac{\left(\left[K_{j}(t)\right]_{n, k}\right)^{2}}{(1+|n|)^{2 \sigma}(1+|k|)^{2 \sigma}} \leq \sup _{n, k \in \mathbb{Z}}\left(\left[K_{j}(t)\right]_{n, k}\right)^{2} b_{j}(t) \leq C t^{-2 \sigma+\varepsilon} \leq C t^{-1} .
$$

In the second case $(n, k) \notin T_{j}$ we have (using $\left.\theta+\pi / 2=\psi\right)$

$$
\begin{aligned}
\left|\left[K_{j}(t)\right]_{n, k}\right| & =\left|\int_{t_{j} \leq|\psi| \leq t_{j+1}} \mathrm{e}^{-\mathrm{i} t(v \psi-2 \sin \psi)} d \psi\right| \\
& =2\left|\int_{t_{j} \leq \psi \leq t_{j+1}} \cos (t(v \psi-2 \sin \psi)) d \psi\right| .
\end{aligned}
$$

Applying integration by parts we get

$$
\begin{aligned}
\left|\left[K_{j}(t)\right]_{n, k}\right| & \leq \frac{2}{t}\left(\frac{1}{\left|v-2 \cos t_{j}\right|}+\frac{1}{\left|v-2 \cos t_{j+1}\right|}+\int_{t_{j}}^{t_{j+1}} \frac{2 \sin (\psi) d \psi}{(v-2 \cos \psi)^{2}}\right) \\
& \leq \frac{4}{t}\left(\frac{1}{\left|4 \sin ^{2}\left(t_{j} / 2\right)+v-2\right|}+\frac{1}{\left|4 \sin ^{2}\left(t_{j+1} / 2\right)+v-2\right|}\right) .
\end{aligned}
$$

Since for $j=1, \ldots, N-1$ we have $|v-2| \geq t_{j} t^{\varepsilon} \gg t_{j+1}^{2}=t_{j}>t_{j}^{2}$, we see

$$
\left|4 \sin ^{2} \frac{t_{j+s}}{2}+v-2\right| \geq|v-2|-4 \sin ^{2} \frac{t_{j+s}}{2} \geq|v-2|-t_{j+s}^{2} \geq|v-2|-t_{j}
$$

for $s=0,1$. But $|v-2|-t_{j} \geq t_{j}\left(t^{\varepsilon}-1\right) \geq C t_{j}$, therefore

$$
\sup _{(n, k) \notin T_{j}}\left|\left[K_{j}(t)\right]_{n, k}\right| \leq C t^{-1} t_{j}^{-1} \leq C t^{-1 / 2}, \quad j=1, \ldots, N-1 .
$$


For $j=N$ we have $|v-2| \geq 1$ and thus

$$
\sin ^{2}\left(\frac{t_{N+1}}{2}\right)=4 \sin ^{2}\left(\frac{\pi}{12}\right)<|v-2| / 2 .
$$

Respectively, $\left|4 \sin ^{2}\left(t_{N+1} / 2\right)+v-2\right| \geq|v-2| / 2$, which implies $\left|\left[K_{N}(t)\right]_{n, k}\right| \leq$ $C t^{-1}$. Combining this with (2.16) we get (2.11) for each $K_{j}(t)$ as $1 \leq j \leq N$.

This finishes the proof of Proposition 2.1.

Remark 2.3. The decay rate in (2.6) is "sharp" as can be seen from the following asymptotics of the Bessel function

$$
J_{t}(t) \sim t^{-1 / 3}, \quad t \rightarrow \infty
$$

see [21, Section 8.2].

\section{Jost solutions and the resolvent}

Consider the Jost solutions $f^{ \pm}(\theta)$ to the equation

$$
H f:=\left(-\Delta_{L}+q\right) f=\omega f
$$

normalized as

$$
f_{n}^{ \pm}(\theta) \sim \mathrm{e}^{\mp \mathrm{i} n \theta}, \quad n \rightarrow \pm \infty,
$$

where $\omega \in \bar{\Xi}$ and $\theta=\theta(\omega) \in \bar{\Sigma}$ (cf. (2.2)). For $q \in \ell_{1}^{1}$ this solution exists everywhere in $\bar{\Xi}$, but for $q \in \ell^{1}$ it exists outside of the edges of continuous spectrum. Introduce

$$
h_{n}^{ \pm}(\theta)=\mathrm{e}^{ \pm \mathrm{i} n \theta} f_{n}^{ \pm}(\theta)
$$

and set

$$
\bar{\Sigma}_{\delta}:=\left\{\theta \in \bar{\Sigma}:\left|\mathrm{e}^{-\mathrm{i} \theta} \pm 1\right|>\delta\right\}, \quad 0<\delta<\sqrt{2} .
$$

Lemma 3.1. (i) Let $q \in \ell_{s}^{1}$ with $s=0,1$, 2. Then the functions $h_{n}^{ \pm}(\theta)$ can be differentiated stimes on $\bar{\Sigma}_{\delta}$, and the following estimates hold:

$$
\left|\frac{\partial^{p}}{\partial \theta^{p}} h_{n}^{ \pm}(\theta)\right| \leq C(\delta) \max \left((\mp n)|n|^{p-1}, 1\right), \quad n \in \mathbb{Z}, 0 \leq p \leq s, \theta \in \bar{\Sigma}_{\delta} .
$$

(ii) If additionally $q \in \ell_{s+1}^{1}$, then $h_{n}^{ \pm}(\theta)$ can be differentiated $s$ times on $\bar{\Sigma}$, and the following estimates hold:

$$
\left|\frac{\partial^{p}}{\partial \theta^{p}} h_{n}^{ \pm}(\theta)\right| \leq C \max \left((\mp n)|n|^{p}, 1\right), \quad n \in \mathbb{Z}, 0 \leq p \leq s, \theta \in \bar{\Sigma} .
$$


Proof. The proof of (3.2) is similar for " + " and " - " cases, hence we give it only for the " + " case. Denote $h_{n}(z)=h_{n}^{+}(\theta)$ with $z=\mathrm{e}^{-\mathrm{i} \theta},|z| \leq 1$. Function $h_{n}(z)$ satisfies the integral equation (see [20])

$$
h_{n}(z)=1+\sum_{m=n+1}^{\infty} G(n, m, z) h_{m}(z), \quad G(n, m, z):=q_{m} \frac{z^{2 m-2 n}-1}{z^{-1}-z} .
$$

For $\theta \in \bar{\Sigma}_{\delta}$ we have $\left|z^{2}-1\right| \geq C(\delta)>0$. Then

$$
|G(n, m, z)| \leq \frac{2|z|\left|q_{m}\right|}{\left|z^{2}-1\right|} \leq C(\delta)\left|q_{m}\right|, \quad m-n>0,
$$

and the method of successive approximations as in [3] implies $\left|h_{n}(z)\right| \leq C(\delta)$. Then (3.2) with $p=0$ follows. Further,

$$
\left|\frac{d^{p}}{d z^{p}} G(n, m, z)\right| \leq C(\delta)(m-n)^{p}\left|q_{m}\right|, \quad p \geq 1, m-n>0, \theta \in \bar{\Sigma}_{\delta} .
$$

Now let $q \in \ell_{1}^{1}$. Consider the first derivative of $h_{n}(z)$. We have

$$
\frac{d}{d z} h_{n}(z)=\phi_{n}(z)+\sum_{m=n+1}^{\infty} G(n, m, z) \frac{d}{d z} h_{m}(z),
$$

where

$$
\phi_{n}(z):=\sum_{m=n+1}^{\infty} h_{m}(z) \frac{d}{d z} G(n, m, z)
$$

with $\left|\phi_{n}(z)\right| \leq C(\delta)$ as $n \geq 0$ and $\theta \in \bar{\Sigma}_{\delta}$ by (3.2) with $p=0$ and (3.5). Applying the method of successive approximations to (3.6) we get (3.2) with $p=1$. For the case $p=2$ we proceed in the same way.

The estimate (3.3) can be obtained from (3.4) by the same approach by virtue of the estimate $\left|\frac{d^{p}}{d z^{p}} G(n, m, z)\right| \leq 2\left|q_{m}\right|(m-n)^{p+1}$, which is valid for all $|z| \leq 1$ and $m>n$.

Corollary 3.2. In the case $q \in \ell^{1}$ Lemma 3.1 (i) implies in particular that for any $\theta \in \bar{\Sigma} \backslash\{0, \pi,-\pi\}$ we got the estimate $\left|h_{n}^{ \pm}(\theta)\right| \leq C(\theta)$ for all $n \in \mathbb{Z}$, where $C(\theta)$ can be chosen uniformly in compact subsets of $\bar{\Sigma}$ avoiding the band edges. Together with (3.1) this implies

$$
\left|f_{n}^{ \pm}(\theta)\right| \leq C(\theta) \mathrm{e}^{ \pm \operatorname{Im}(\theta) n}, \quad \theta \in \bar{\Sigma} \backslash\{0, \pi,-\pi\}, n \in \mathbb{Z} .
$$


Given the Jost solutions we can express the kernel of the resolvent

$$
\mathcal{R}(\omega)=(H-\omega)^{-1}: \ell^{2} \longrightarrow \ell^{2}
$$

for $\omega \in \mathbb{C} \backslash \operatorname{spec}(H)$ as (cf. [20, (1.99)])

$$
[\mathcal{R}(\omega)]_{n, k}=\frac{1}{W(\theta(\omega))} \begin{cases}f_{n}^{+}(\theta(\omega)) f_{k}^{-}(\theta(\omega)) & \text { for } n \geq k, \\ f_{k}^{+}(\theta(\omega)) f_{n}^{-}(\theta(\omega)) & \text { for } n \leq k,\end{cases}
$$

where

$$
W(\theta):=W\left(f^{+}(\theta), f^{-}(\theta)\right)=f_{0}^{+}(\theta) f_{1}^{-}(\theta)-f_{1}^{+}(\theta) f_{0}^{-}(\theta)
$$

is the Wronskian of the Jost solutions. Recall that $\theta \mapsto \omega(\theta)$ is a biholomorphic map $\Sigma \rightarrow \Xi$.

The representation (3.8), the fact that $W(\theta)$ does not vanish for $\omega \in(0,4)$, and the bound (3.7) imply the limiting absorption principle for the perturbed onedimensional Schrödinger equation.

Lemma 3.3. Let $q \in \ell^{1}$. Then the convergence

$$
\mathcal{R}(\omega \pm \mathrm{i} \varepsilon) \longrightarrow \mathcal{R}(\omega \pm \mathrm{i} 0), \quad \varepsilon \rightarrow 0+, \omega \in(0,4)
$$

holds in $\mathcal{L}\left(\ell_{\sigma}^{2}, \ell_{-\sigma}^{2}\right)$ with $\sigma>1 / 2$.

Proof. For any $\omega \in(0,4)$ and any $n, k \in \mathbb{Z}$, there exist the pointwise limit

$$
[\mathcal{R}(\omega \pm \mathrm{i} \varepsilon)]_{n, k} \longrightarrow[\mathcal{R}(\omega \pm \mathrm{i} 0)]_{n, k}, \quad \varepsilon \rightarrow 0 .
$$

Moreover, the bound (3.7) implies that $\left|[\mathcal{R}(\omega \pm \mathrm{i} \varepsilon)]_{n, k}\right| \leq C(\omega)$. Hence, the Hilbert-Schmidt norm of the difference $\mathcal{R}(\omega \pm \mathrm{i} \varepsilon)-\mathcal{R}(\omega \pm \mathrm{i} 0)$ converges to zero in $B(\sigma,-\sigma)$ with $\sigma>1 / 2$ by the Lebesgue dominated convergence theorem.

Corollary 3.4. For any $\omega \in(0,4)$ and any fixed $\sigma>1 / 2$, the operators

$$
\mathcal{R}(\omega \pm i 0): \ell_{\sigma}^{2} \longrightarrow \ell_{-\sigma}^{2}
$$

have integral kernels given by

$$
[\mathcal{R}(\omega \pm i 0)]_{n, k}=\frac{1}{W\left(\theta_{ \pm}\right)} \begin{cases}f_{n}^{+}\left(\theta_{ \pm}\right) f_{k}^{-}\left(\theta_{ \pm}\right) & \text {for } n \geq k, \\ f_{k}^{+}\left(\theta_{ \pm}\right) f_{n}^{-}\left(\theta_{ \pm}\right) & \text {for } n \leq k,\end{cases}
$$

where $\theta_{+}$, and $\theta_{-}=-\theta_{+}$are defined by (2.4). 
At the end of this section we discuss an alternative definition of resonances.

Definition 3.5. For $\omega \in\{0,4\}$ any nonzero solution $u \in \ell^{\infty}(\mathbb{Z})$ of the equation $H u=\omega u$ is called a resonance function, and in this case the point $\omega$ is called a resonance.

Lemma 3.6. Let $q \in \ell_{1}^{1}$. Then $\omega=0($ or $\omega=4)$ is a resonance if and only if $W(0)=0($ or $W(\pi)=0)$.

Proof. We consider the case $\omega=0$. In this case $f_{n}^{ \pm}=1+o(1)$, as $n \rightarrow \pm \infty$. Introduce another solution $g^{+}$satisfying $W\left(f^{+}, g^{+}\right)=1$. Making the ansatz $g_{n}^{+}=f_{n}^{+} v_{n}$, where $v_{n}$ is unknown, we obtain $\left(v_{n+1}-v_{n}\right) f_{n}^{+} f_{n+1}^{+}=1$ for sufficiently large positive $n_{0}$. Solving for $v$ shows

$$
g_{n}^{+}=f_{n}^{+} \sum_{j=n_{0}}^{n-1} \frac{1}{f_{j}^{+} f_{j+1}^{+}}+v_{n_{0}} f_{n}^{+}=n+o(n), \quad n \rightarrow+\infty .
$$

Hence $f_{n}^{-}=\alpha f_{n}^{+}+\beta g_{n}^{+}$and there is a bounded solution if and only if $\beta=W\left(f^{+}, f^{-}\right)=0$.

\section{Properties of the scattering matrix}

Recall that the Wiener algebra is the set of all integrable functions whose Fourier coefficients are integrable:

$$
\mathcal{A}=\left\{f(\theta)=\sum_{m \in \mathbb{Z}} \hat{f}_{m} \mathrm{e}^{\mathrm{i} m \theta} \mid\|\hat{f}\|_{\ell^{1}}<\infty\right\} .
$$

We set

$$
\|f\|_{\mathcal{A}}=\|\hat{f}\|_{\ell^{1}} .
$$

In the case $q \in \ell_{1}^{1}$ the functions $h_{n}^{ \pm}$from (3.1) can be represented as

$$
h_{n}^{ \pm}(\theta)=1+\sum_{m= \pm 1}^{ \pm \infty} B_{n, m}^{ \pm} \mathrm{e}^{\mp \mathrm{i} m \theta}
$$


where (see [20, Sect. 10.1]) $B_{n, m}^{ \pm} \in \mathbb{R}$ and

$$
\left|B_{n, m}^{ \pm}\right| \leq C_{n}^{ \pm} \sum_{k=n+\lfloor m / 2\rfloor}^{ \pm \infty}\left|q_{k}\right|,
$$

with

$$
C_{n}^{ \pm} \leq C^{ \pm}, \quad \pm n \geq \mp 1 .
$$

The estimate (4.3) implies

$$
h_{n}^{ \pm}(\theta), f_{n}^{ \pm}(\theta) \in \mathcal{A} \quad \text { if } q \in \ell_{1}^{1} .
$$

Moreover, the Wronskian $W(\theta)$ (see (3.9)) of Jost solutions also belongs to the Wiener algebra $\mathcal{A}$ if $q \in \ell_{1}^{1}$ and the same holds true for the Wronskians

$$
W^{ \pm}(\theta)=W\left(f^{\mp}(\theta), f^{ \pm}(-\theta)\right) .
$$

Moreover, we have the scattering relations

$$
T(\theta) f_{m}^{ \pm}(\theta)=R^{\mp}(\theta) f_{m}^{\mp}(\theta)+f_{m}^{\mp}(-\theta), \quad \theta \in[-\pi, \pi],
$$

where the quantities

$$
T(\theta)=\frac{2 \mathrm{i} \sin \theta}{W(\theta)}, \quad R^{ \pm}(\theta)= \pm \frac{W^{ \pm}(\theta)}{W(\theta)},
$$

which are known as the transmission and reflection coefficients, also belong to this algebra:

Theorem 4.1. If $q \in \ell_{1}^{1}$, then $T(\theta), R^{ \pm}(\theta) \in \mathcal{A}$.

Proof. The Wronskian $W(\theta)$ can vanish only at the edges of continuous spectra, i.e. when $\theta=0, \pm \pi$, which correspond to the resonant cases (see Lemma 3.6 below). Remind that we identify points $\pi$ and $-\pi$, considering Jost solutions, Wronskians and scattering data as functions on the unit circle. Thus it is sufficient to consider the points 0 and $\pi$. Since $|T(\theta)| \leq 1$ as $\theta \in[-\pi, \pi]$ then the zeros of the Wronskian at points $0, \pi$ can be at most of first order. Since $W(\theta) \in \mathcal{A}$ by (4.5), then in the case $W(0) W(\pi) \neq 0$ we obtain $W(\theta)^{-1} \in \mathcal{A}$ by Wiener's lemma. Therefore, $T, R^{ \pm} \in \mathcal{A}$.

If $W(0) W(\pi)=0$ we need to work a bit harder. Suppose, for example, $W(0)=0$. In [4], Lemma 4.1, formulas (4.12)-(4.14), the following representation is obtained

$$
V^{ \pm}(\theta):=f_{1}^{ \pm}(\theta) f_{0}^{ \pm}(0)-f_{0}^{ \pm}(\theta) f_{1}^{ \pm}(0)=\left(1-\mathrm{e}^{\mathrm{i} \theta}\right) \Psi^{ \pm}(\theta),
$$


where

$$
\Psi^{ \pm}(\theta)=\sum_{l=\frac{1 \pm 1}{2}}^{ \pm \infty} g_{m}^{ \pm} \mathrm{e}^{\mp \mathrm{i} m \theta}, \quad \text { with } g^{ \pm} \in \ell^{1}\left(\mathbb{Z}_{ \pm}\right) \text {if } q \in \ell_{1}^{1}
$$

In other words, $\Psi^{ \pm}(\theta) \in \mathcal{A}$. Since

$$
W(0)=f_{0}^{+}(0) f_{1}^{-}(0)-f_{1}^{+}(0) f_{0}^{-}(0)=0
$$

we have two possible combinations (since the solutions $f_{m}^{ \pm}(0)$ cannot vanish at two consecutive points): (a) $f_{0}^{+}(0) f_{0}^{-}(0) \neq 0$ and (b) $f_{1}^{+}(0) f_{1}^{-}(0) \neq 0$. Consider the case (a). By (3.9), (4.8), and (4.10) we get

$$
\begin{aligned}
W(\theta) & =f_{0}^{+}(\theta) f_{0}^{-}(\theta)\left(\frac{V^{-}(\theta)}{f_{0}^{-}(0) f_{0}^{-}(\theta)}-\frac{V^{+}(\theta)}{f_{0}^{+}(0) f_{0}^{+}(\theta)}\right) \\
& =\left(1-\mathrm{e}^{\mathrm{i} \theta}\right)\left(\frac{f_{0}^{+}(\theta)}{f_{0}^{-}(0)} \Psi^{-}(\theta)-\frac{f_{0}^{-}(\theta)}{f_{0}^{+}(0)} \Psi^{+}(\theta)\right) \\
& =\left(1-\mathrm{e}^{\mathrm{i} \theta}\right) \Phi(\theta),
\end{aligned}
$$

where $\Phi(\theta) \in \mathcal{A}$ by (4.9) and (4.5). We observe that if $W(\pi)=0$ then $\Phi(\theta) \neq 0$ for $\theta \in(-\pi, \pi)$ and if $W(\pi) \neq 0$ then $\Phi(\theta) \neq 0$ for $\theta \in[-\pi, \pi]$. The same result follows in a similar fashion in case (b). Since equality $W(0)=0$ implies $W^{ \pm}(0)=0$ then we can also get similarly

$$
W^{ \pm}(\theta)=\left(1-\mathrm{e}^{\mathrm{i} \theta}\right) \Phi^{ \pm}(\theta)
$$

with $\Phi^{ \pm}(\theta) \in \mathcal{A}$.

Analogously, $W(\pi)=0$ implies

$$
W(\theta)=\left(1+\mathrm{e}^{\mathrm{i} \theta}\right) \widetilde{\Phi}(\theta), \quad W^{ \pm}(\theta)=\left(1+\mathrm{e}^{\mathrm{i} \theta}\right) \widetilde{\Phi}^{ \pm}(\theta)
$$

with $\widetilde{\Phi}, \widetilde{\Phi}^{ \pm} \in \mathcal{A}$ and $\widetilde{\Phi}(\theta) \neq 0$ for $\theta \in[-\pi, \pi]$ if $W(0) \neq 0$. Thus if $W$ vanishes at only one edge of spectrum, this finishes the proof. If $W$ vanishes at both edges, then we can use a smooth cut-off function to combine both representations into $W(\theta)=\left(1-\mathrm{e}^{2 \mathrm{i} \theta}\right) \breve{\Phi}(\theta)$ (respectively, $\left.W^{ \pm}(\theta)=\left(1-\mathrm{e}^{2 \mathrm{i} \theta}\right) \breve{\Phi}^{ \pm}(\theta)\right)$ with $\breve{\Phi}, \breve{\Phi}^{ \pm} \in \mathcal{A}$ and $\breve{\Phi}(\theta) \neq 0$ for $\theta \in[-\pi, \pi]$. 


\section{Dispersive decay in the resonant case}

We begin with a small variant of the van der Corput lemma.

Lemma 5.1. Consider the oscillatory integral

$$
I(t)=\int_{a}^{b} \mathrm{e}^{\mathrm{i} t \phi(\theta)} f(\theta) d \theta, \quad-\pi \leq a<b \leq \pi,
$$

where $\phi(\theta)$ is real-valued. If $\min _{\theta \in[a, b]}\left|\phi^{(s)}(\theta)\right|=m_{s}>0$ for some $s \geq 2$ and $f \in \mathcal{A}$, then

$$
|I(t)| \leq \frac{C_{s}\|\hat{f}\|_{\ell^{1}}}{\left(m_{s} t\right)^{1 / s}}, \quad t \geq 1,
$$

where $C_{s}$ is a universal constant.

Proof. We rewrite

$$
\begin{aligned}
I(t) & =\int_{a}^{b} \mathrm{e}^{\mathrm{i} t \phi(\theta)} \sum_{p \in \mathbb{Z}} \hat{f}_{p} \mathrm{e}^{\mathrm{i} p \theta} d \theta=\sum_{p \in \mathbb{Z}} \hat{f}_{p} I_{p / t}(t), \\
I_{v}(t) & =\int_{a}^{b} \mathrm{e}^{\mathrm{i} t(\phi(\theta)+v \theta)} d \theta .
\end{aligned}
$$

By the van der Corput lemma [19, page 332] we have $\left|I_{v}(t)\right| \leq C_{s}\left(m_{s} t\right)^{-1 / s}$, where $C_{s}$ is a universal constant (independent of $v$ ) and the claim follows.

Remark 5.2. The above lemma is usually found for the case when $f$ is absolutely continuous in the literature (cf. [19, page 333]) — in fact, the proof immediately extends to functions of bounded variation. However, by the Riemann-Lebesgue lemma the Fourier coefficients of an absolutely continuous function must satisfy $\hat{f}_{m}=o\left(m^{-1}\right)$ (for functions of bounded variation one has $O\left(m^{-1}\right)$ ) and considering lacunary Fourier coefficients one obtains an element in the Wiener algebra which is not absolutely continuous (of bounded variation). Conversely, since the Fourier coefficients of an integrable function can have arbitrary slow decay, there are absolutely continuous functions which are not in the Wiener algebra. Finally, note that for continuous $f$ the decay can be arbitrary slow.

Now we come to our main result in this section.

Theorem 5.3. Let $q \in \ell_{1}^{1}$. Then the asymptotics (1.3) and (1.8) hold, i.e.,

$$
\begin{aligned}
\left\|\mathrm{e}^{-\mathrm{i} t H} P_{c}\right\|_{\ell^{1} \rightarrow \ell^{\infty}} & =\mathcal{O}\left(t^{-1 / 3}\right), \quad t \rightarrow \infty, \\
\left\|\mathrm{e}^{-\mathrm{i} t H} P_{c}\right\|_{\ell_{\sigma}^{2} \rightarrow \ell_{-\sigma}^{2}} & =\mathcal{O}\left(t^{-1 / 2}\right), \quad t \rightarrow \infty, \sigma>1 / 2 .
\end{aligned}
$$


Proof. STEP I. We apply the spectral representation

$$
\mathrm{e}^{-\mathrm{i} t H} P_{c}=\frac{1}{2 \pi \mathrm{i}} \int_{[0,4]} \mathrm{e}^{-\mathrm{i} t \omega}(\mathcal{R}(\omega+\mathrm{i} 0)-\mathcal{R}(\omega-\mathrm{i} 0)) d \omega .
$$

Expressing the kernel of the resolvent in terms of the Jost solutions (cf. [20, (1.99)]), the kernel of $\mathrm{e}^{-\mathrm{i} t H} P_{c}$ reads:

$$
\begin{aligned}
{\left[\mathrm{e}^{-\mathrm{i} t H} P_{c}\right]_{n, k} } & =\frac{1}{2 \pi \mathrm{i}} \int_{0}^{4} \mathrm{e}^{-\mathrm{i} t \omega}\left[\frac{f_{k}^{+}\left(\theta_{+}\right) f_{n}^{-}\left(\theta_{+}\right)}{W\left(\theta_{+}\right)}-\frac{f_{k}^{+}\left(\theta_{-}\right) f_{n}^{-}\left(\theta_{-}\right)}{W\left(\theta_{-}\right)}\right] d \omega \\
& =-\frac{1}{\pi \mathrm{i}} \int_{-\pi}^{\pi} \mathrm{e}^{-\mathrm{i} t(2-2 \cos \theta)} \frac{f_{k}^{+}(\theta) f_{n}^{-}(\theta)}{W(\theta)} \sin \theta d \theta
\end{aligned}
$$

for $n \leq k$ and by symmetry $\left[\mathrm{e}^{-\mathrm{i} t H} P_{c}\right]_{n, k}=\left[\mathrm{e}^{-\mathrm{i} t H} P_{c}\right]_{k, n}$ for $n \geq k$. Hence, for (5.3) it suffices to prove that

$$
\left[\mathrm{e}^{-\mathrm{i} t H} P_{c}\right]_{n, k}=\mathcal{O}\left(t^{-1 / 3}\right), \quad t \rightarrow \infty .
$$

independent of $n, k$. We suppose $n \leq k$ for notational simplicity. Then

$$
\left[\mathrm{e}^{-\mathrm{i} t H} P_{c}\right]_{n, k}=\frac{1}{2 \pi} \int_{-\pi}^{\pi} \mathrm{e}^{-\mathrm{i} t \phi_{v}(\theta)} h_{k}^{+}(\theta) h_{n}^{-}(\theta) T(\theta) d \theta,
$$

where $\phi_{v}$ is defined in (2.9) with $v=\frac{k-n}{t} \geq 0$. We observe that the function

$$
Y_{n, k}(\theta)=h_{k}^{+}(\theta) h_{n}^{-}(\theta) T(\theta)
$$

belongs to $\mathcal{A}$, moreover, the $\ell^{1}$-norm of its Fourier coefficients $\hat{Y}_{n, k}(\cdot)$ can be estimated by a value, which does not depend on $n$ and $k$. To this end introduce

$$
1+\sup _{ \pm n>0} \sum_{m=1}^{ \pm \infty}\left|B_{n, m}^{ \pm}\right|=\tilde{C}^{ \pm}>0 .
$$

By (4.3) and (4.4) this supremum is finite. Then

$$
\left\|\hat{h}_{n}^{ \pm}(\cdot)\right\|_{\ell^{1}} \leq \widetilde{C}^{ \pm} \quad \text { for } \pm n>0 .
$$

Now consider the three possibilities:
(a) $n \leq k \leq 0$,
(b) $0 \leq n \leq k$, and
(c) $n \leq 0 \leq k$. 
In the case (c) the bound (5.9) and Theorem 4.1 imply

$$
\left\|\hat{Y}_{n, k}(\cdot)\right\|_{\ell^{1}} \leq C .
$$

In the other two cases we use the scattering relations (4.6) to get the representation

$$
Y_{n, k}(\theta)= \begin{cases}h_{n}^{-}(\theta)\left(R^{-}(\theta) h_{k}^{-}(\theta) \mathrm{e}^{2 \mathrm{i} k \theta}+h_{k}^{-}(-\theta)\right) & n \leq k \leq 0, \\ h_{k}^{+}(\theta)\left(R^{+}(\theta) h_{n}^{+}(\theta) \mathrm{e}^{-2 \mathrm{i} n \theta}+h_{n}^{+}(-\theta)\right) & 0 \leq n \leq k,\end{cases}
$$

and again apply Theorem 4.1 together with (4.5) and (5.9) to obtain (5.10).

Now, as in the proof of (2.6) (see Step I in the proof of Proposition 2.1) we split the domain of integration into regions where either the second or third derivative of the phase is nonzero and apply Lemma 5.1 together with the estimates from Theorem 4.1.

Step in. Set

$$
J:=\left\{\theta \in[-\pi, \pi]:\left|\theta \pm \frac{\pi}{2}\right| \leq \frac{\pi}{6}\right\} .
$$

To establish (5.4) we represent $\left[\mathrm{e}^{-\mathrm{i} t H} P_{c}\right]_{n, k}$ as the sum

$$
\left[\mathrm{e}^{-\mathrm{i} t H} P_{c}\right]_{n, k}=\left[\mathcal{K}^{ \pm}(t)\right]_{n, k}+[\tilde{\mathcal{K}}(t)]_{n, k},
$$

where

$$
\begin{aligned}
{\left[\mathcal{K}^{ \pm}(t)\right]_{n, k} } & =\frac{1}{2 \pi} \int_{\left|\theta \pm \frac{\pi}{2}\right| \leq \frac{\pi}{6}} \mathrm{e}^{-\mathrm{i} t \phi_{v}(\theta)} Y_{n, k}(\theta) d \theta, \\
{[\tilde{\mathcal{K}}(t)]_{n, k} } & =\frac{1}{2 \pi} \int_{\theta \in[-\pi, \pi] \backslash J} \mathrm{e}^{-\mathrm{i} t \phi_{v}(\theta)} Y_{n, k}(\theta) d \theta,
\end{aligned}
$$

and $Y_{n, k}(\theta)=h_{k}^{+}(\theta) h_{n}^{-}(\theta) T(\theta)$ as above. Lemma 5.1 with $s=2$ and the bound, equation (5.10) imply

$$
\sup _{n, k \in \mathbb{Z}}\left|[\tilde{\mathcal{K}}(t)]_{n, k}\right| \leq C t^{-1 / 2}, \quad t \geq 1 .
$$

Then

$$
\|\widetilde{\mathcal{K}}(t)\|_{\ell_{\sigma}^{2} \rightarrow \ell_{-\sigma}^{2}} \leq C t^{-1 / 2}, \quad \sigma>1 / 2, t \geq 1 .
$$

It remains to obtain the same estimate for $\mathcal{K}^{ \pm}(t)$. Since $W(\theta) \neq 0$ for $\theta \in J$, it follows from Lemma 3.1 that

$$
\left|\frac{d}{d \theta} T(\theta)\right|, \quad\left|\frac{d}{d \theta} R^{ \pm}(\theta)\right| \leq C, \quad \theta \in J .
$$


Furthermore, we split $\mathcal{K}^{ \pm}(t)$ as

$$
\mathcal{K}^{ \pm}(t)=\mathcal{K}_{a}^{ \pm}(t)+\mathcal{K}_{b}^{ \pm}(t)+\mathcal{K}_{c}^{ \pm}(t),
$$

where $\mathcal{K}_{a}^{ \pm}(t)$ are the restrictions of the operators $\mathcal{K}^{ \pm}(t)$ to the case (a) $n \leq k \leq 0$ etc. First we estimate $\mathcal{K}_{c}^{ \pm}(t)$. The bounds (3.2) and (5.12) imply

$$
\left|\frac{\partial}{\partial \theta} Y_{n, k}(\theta)\right| \leq C, \quad \theta \in J, n \leq 0 \leq k .
$$

Therefore, applying integration by parts, we obtain

$$
\left|\left[\mathcal{K}_{c}^{-}(t)\right]_{n, k}\right| \leq C t^{-1}, \quad t \geq 1,
$$

and then

$$
\left\|\mathcal{K}_{c}^{-}(t)\right\|_{\ell_{\sigma}^{2} \rightarrow \ell_{-\sigma}^{2}} \leq C t^{-1}, \quad \sigma>1 / 2, t \geq 1 .
$$

To estimate $\mathcal{K}_{c}^{+}(t)$ we apply the general scheme of Lemma 2.2. In particular, to prove a bound of the type (2.13) for the integral with the additional factor $Y_{n, k}$ we use Lemma 5.1. To get (2.17) we use the bounds (5.10) and (5.13). For the other estimates we repeat literally the respective estimates of Lemma 2.2. Thus, we obtain

$$
\left\|\mathcal{K}_{c}^{+}(t)\right\|_{\ell_{\sigma}^{2} \rightarrow \ell_{-\sigma}^{2}} \leq C t^{-1 / 2}, \quad \sigma>1 / 2, t \rightarrow \infty .
$$

Now consider the case (a). Using the first line of (5.11) and the fact

$$
\mathrm{i}(n-k)+2 \mathrm{i} k=\mathrm{i}(k+n)=-\mathrm{i}|k+n|, \quad n \leq k \leq 0,
$$

we represent $\mathcal{K}_{a}^{ \pm}(t)$ as

$$
\mathcal{K}_{a}^{ \pm}(t)=\frac{1}{2 \pi} \int_{\left|\theta \pm \frac{\pi}{2}\right| \leq \frac{\pi}{6}} \mathrm{e}^{-\mathrm{i} t \phi_{v}(\theta)} Y_{n, k}^{1}(\theta) d \theta+\frac{1}{2 \pi} \int_{\left|\theta \pm \frac{\pi}{2}\right| \leq \frac{\pi}{6}} \mathrm{e}^{-\mathrm{i} t \tilde{\phi}_{v}(\theta)} Y_{n, k}^{2}(\theta) d \theta
$$

where

$$
\begin{aligned}
& Y_{n, k}^{1}(\theta)=h_{n}^{-}(\theta) h_{k}^{-}(-\theta), \\
& Y_{n, k}^{2}(\theta)=R^{-}(\theta) h_{n}^{-}(\theta) h_{k}^{-}(\theta),
\end{aligned}
$$

and

$$
\tilde{\phi}_{v}(\theta)=2-2 \cos \theta+\tilde{v} \theta, \quad \text { with } \tilde{v}=|n+k| / t \geq 0 \text {. }
$$

Since

$$
\left|\frac{\partial}{\partial \theta} Y_{n, k}^{j}(\theta)\right| \leq C, \quad \theta \in J, n \leq k \leq 0, j=1,2,
$$

then $\mathcal{K}_{a}^{ \pm}(t)$ can be treated similarly to $\mathcal{K}_{c}^{ \pm}(t)$.

In the case (b) we have

$$
\mathrm{i}(n-k)-2 \mathrm{i} n=-\mathrm{i}(k+n)=-\mathrm{i}|k+n|, \quad 0 \leq n \leq k .
$$

and then the proof is the same as in the case (a). 


\section{Dispersive decay in the non-resonant case}

Theorem 6.1. Let $q \in \ell_{2}^{1}$. Then in the non-resonant case the asymptotics (1.9) hold, i.e.,

$$
\left\|\mathrm{e}^{-\mathrm{i} t H} P_{c}\right\|_{\ell_{1}^{1} \rightarrow \ell_{-1}^{\infty}}=\mathcal{O}\left(t^{-4 / 3}\right), \quad t \rightarrow \infty,
$$

Proof. It suffices to show that

$$
\left|\left[\mathrm{e}^{-\mathrm{i} t H} P_{c}\right]_{n, k}\right| \leq C(1+|n|)(1+|k|) t^{-4 / 3}, \quad t \geq 1 .
$$

The representation (4.2) and the bounds (4.3)-(4.4) imply

$$
h_{n}^{ \pm}(\theta), \quad \frac{\partial}{\partial \theta} h_{n}^{ \pm}(\theta) \in \mathcal{A} \quad \text { if } q \in \ell_{2}^{1} .
$$

Therefore, $\frac{d}{d \theta} W(\theta):=W^{\prime}(\theta) \in \mathcal{A}$. Since in the non-resonant case $W(\theta)^{-1} \in \mathcal{A}$ we also infer

$$
\frac{d}{d \theta} T(\theta), \quad \frac{d}{d \theta} R^{ \pm}(\theta) \in \mathcal{A}
$$

by Wiener's lemma. For the derivatives of $h_{k}^{ \pm}$bounds of the type (5.9) hold, namely,

$$
\left\|\frac{\partial}{\partial \theta} h_{n}^{ \pm}(\cdot)\right\|_{\mathcal{A}} \leq \widetilde{C} \quad \text { for } \pm n>0 .
$$

For $n \leq k$ we represent the jump of the resolvent across the spectrum as

$\mathcal{R}(\omega+\mathrm{i} 0)-\mathcal{R}(\omega-\mathrm{i} 0))=\frac{T(\theta) f_{k}^{+}(\theta) f_{n}^{-}(\theta)+\overline{T(\theta) f_{k}^{+}(\theta)} \overline{f_{n}^{-}(\theta)}}{-2 \mathrm{i} \sin \theta}, \quad \theta \in[0, \pi]$.

The scattering relations (4.6) imply

$$
\begin{aligned}
& f_{n}^{-}(\theta)=T(-\theta) f_{n}^{+}(-\theta)-R^{-}(-\theta) f_{n}^{-}(-\theta), \\
& \overline{f_{k}^{+}(\theta)}=T(\theta) f_{k}^{-}(\theta)-R^{+}(\theta) f_{k}^{+}(\theta) .
\end{aligned}
$$

Then using the consistency relation $T \overline{R^{-}}+\bar{T} R^{+}=0$ we come to the formula (cf. [17, p.13])

$$
\mathcal{R}(\omega+\mathrm{i} 0)-\mathcal{R}(\omega-\mathrm{i} 0))=\frac{|T(\theta)|^{2}}{-2 \mathrm{i} \sin \theta}\left[f_{k}^{+}(\theta) f_{n}^{+}(-\theta)+f_{k}^{-}(\theta) f_{n}^{-}(-\theta)\right], \quad \theta \in[0, \pi] .
$$


Inserting this into (5.5) and integrating by parts we get

$$
\begin{aligned}
& {\left[\mathrm{e}^{-\mathrm{i} t H} P_{c}\right]_{n, k}} \\
& \quad=\frac{1}{\pi} \int_{-\pi}^{\pi} \mathrm{e}^{-\mathrm{i} t(2-2 \cos \theta)}|T(\theta)|^{2}\left[f_{k}^{+}(\theta) f_{n}^{+}(-\theta)+f_{k}^{-}(\theta) f_{n}^{-}(-\theta)\right] d \theta \\
& \quad=\frac{\mathrm{i}}{2 \pi t} \int_{-\pi}^{\pi} \mathrm{e}^{-\mathrm{i} t(2-2 \cos \theta)} \frac{d}{d \theta}\left[\frac{|T(\theta)|^{2}}{\sin \theta}\left(f_{k}^{+}(\theta) f_{n}^{+}(-\theta)+f_{k}^{-}(\theta) f_{n}^{-}(-\theta)\right)\right] d \theta \\
& \quad=\left[\mathrm{e}^{-\mathrm{i} t H} P_{c}\right]_{n, k}^{+}+\left[\mathrm{e}^{-\mathrm{i} t H} P_{c}\right]_{n, k}^{-} .
\end{aligned}
$$

Evaluating the derivative we further obtain

$$
\begin{aligned}
{\left[\mathrm{e}^{-\mathrm{i} t H} P_{c}\right]_{n, k}^{ \pm}=} & \frac{\mathrm{i}}{2 \pi t} \int_{-\pi}^{\pi} \mathrm{e}^{-\mathrm{i} t(2-2 \cos \theta)} \frac{d}{d \theta}\left[\frac{|T(\theta)|^{2}}{\sin \theta} \mathrm{e}^{\mp \mathrm{i} \theta(k-n)} h_{k}^{ \pm}(\theta) h_{n}^{ \pm}(-\theta)\right] d \theta \\
= & \frac{ \pm(k-n)}{2 \pi t} \int_{-\pi}^{\pi} \mathrm{e}^{-\mathrm{i} t(2-2 \cos \theta)} \mathrm{e}^{\mp \mathrm{i} \theta(k-n)} \frac{|T(\theta)|^{2}}{\sin \theta} h_{k}^{ \pm}(\theta) h_{n}^{ \pm}(-\theta) d \theta \\
& -\frac{\mathrm{i}}{2 \pi t} \int_{-\pi}^{\pi} \mathrm{e}^{-\mathrm{i} t(2-2 \cos \theta)} \mathrm{e}^{\mp \mathrm{i} \theta(k-n)} \cos \theta \frac{|T(\theta)|^{2}}{\sin ^{2} \theta} h_{k}^{ \pm}(\theta) h_{n}^{ \pm}(-\theta) d \theta \\
& +\frac{\mathrm{i}}{2 \pi t} \int_{-\pi}^{\pi} \mathrm{e}^{-\mathrm{i} t(2-2 \cos \theta)} \mathrm{e}^{\mp \mathrm{i} \theta(k-n)} \frac{\frac{d}{d \theta}\left[|T(\theta)|^{2} h_{k}^{ \pm}(\theta) h_{n}^{ \pm}(-\theta)\right]}{\sin \theta} d \theta .
\end{aligned}
$$

Next, observe that formula (4.3) implies that if $q \in \ell_{2}^{1}$, then $B_{m, s}^{ \pm} \in \ell_{1}^{1}\left(\mathbb{Z}_{ \pm}\right)$for any fixed $m$, and consequently

$$
S_{m}^{ \pm}(j):=\sum_{s=j}^{ \pm \infty}\left|B_{m, s}^{ \pm}\right|, \quad S_{m}^{ \pm}(\cdot) \in \ell^{1}\left(\mathbb{Z}_{ \pm}\right) .
$$

Based on this observation we prove the following

Lemma 6.2. Let $q \in \ell_{2}^{1}$ and $W(0) W(\pi) \neq 0$. Then $T(\theta) h_{m}^{ \pm}(\theta) / \sin \theta \in \mathcal{A}$, and

$$
\left\|\frac{T(\theta) h_{m}^{ \pm}(\theta)}{\sin \theta}\right\|_{\mathcal{A}} \leq C(1+|m|), \quad m \in \mathbb{Z} .
$$

Proof. Since $T(\theta) / \sin \theta=2 \mathrm{i} / W(\theta)$ then for $m \in \mathbb{Z}_{ \pm}$the bound (6.8) follows from (5.9) and Theorem 4.1. Hence it remains to consider the case $m \in \mathbb{Z}_{\mp}$. The scattering relations (4.6) imply

$$
\begin{aligned}
T(\theta) h_{m}^{ \pm}(\theta) & =\left(R^{\mp}(\theta)+1\right) h_{m}^{\mp}(\theta) \mathrm{e}^{ \pm 2 \mathrm{i} m \theta} \\
& -\left(h_{m}^{\mp}(\theta)-h_{m}^{\mp}(-\theta)\right) \mathrm{e}^{ \pm 2 \mathrm{i} m \theta} \\
& +h_{m}^{\mp}(-\theta)\left(1-\mathrm{e}^{ \pm 2 \mathrm{i} m \theta}\right) .
\end{aligned}
$$


Using (4.2) we obtain

$$
\begin{aligned}
\frac{h_{m}^{\mp}(\theta)-h_{m}^{\mp}(-\theta)}{\sin \theta} & =\sum_{s=\mp 1}^{\mp \infty} B_{m, s}^{\mp} \frac{\mathrm{e}^{\mp \mathrm{i} s \theta}-\mathrm{e}^{ \pm \mathrm{i} s \theta}}{\sin \theta} \\
& =\mp 2 \mathrm{i} \sum_{s=\mp 1}^{\mp \infty} B_{m, s}^{\mp} \sum_{j=-(s-1)}^{s-1} \mathrm{e}^{\mathrm{i} j \theta} \\
& =\mp 2 \mathrm{i} \sum_{j=-\infty}^{\infty}\left(\sum_{s=\mp|j| \mp 1}^{\mp \infty} B_{j, s}^{\mp}\right) \mathrm{e}^{\mathrm{i} j \theta} .
\end{aligned}
$$

Property (6.7) then implies

$$
\left\|\frac{h_{m}^{\mp}(\theta)-h_{m}^{\mp}(-\theta)}{\sin \theta}\right\|_{\mathcal{A}} \leq C, \quad m \in \mathbb{Z}_{\mp} .
$$

and we get

$$
\frac{f_{0}^{\mp}(\theta)-f_{0}^{\mp}(-\theta)}{\sin \theta}, \quad \frac{f_{1}^{+}(\theta)-f_{1}^{+}(-\theta)}{\sin \theta}, \quad \frac{f_{-1}^{-}(\theta)-f_{-1}^{-}(-\theta)}{\sin \theta} \in \mathcal{A}, \quad q \in \ell_{2}^{1},
$$

as well as

$$
\frac{R^{\mp}(\theta)+1}{\sin \theta}=\frac{1}{W(\theta)} \frac{W(\theta) \mp W^{\mp}(\theta)}{\sin \theta} \in \mathcal{A} .
$$

Furthermore,

$$
\left\|\frac{1-\mathrm{e}^{ \pm 2 \mathrm{i} m \theta} \theta}{\sin \theta}\right\|_{\mathcal{A}} \leq 2|m| .
$$

Finally, substituting (6.10), (6.11), and (6.12) into (6.9) we get (6.8).

To obtain (6.2) for the first summand in (6.6) note that $k-n \leq 2 \max \{|n|,|k|\}$. Hence we apply (6.8) to the factor $T(-\theta) h_{m}^{ \pm}(-\theta) / \sin \theta$, where $|m|=\min \{|n|,|k|\}$. Then we split the domain of integration into regions where either the second or third derivative of the phase is nonzero and apply Lemma 5.1 together with estimate from Theorem 4.1 and Lemma 6.2. To obtain (6.2) for the second summand in (6.6) we apply (6.8) to both $T(-\theta) h_{n}^{ \pm}(-\theta) / \sin \theta$ and $T(\theta) h_{k}^{ \pm}(\theta) / \sin \theta$.

To complete the proof of (6.1) we need one more property. 
Lemma 6.3. Let $q \in \ell_{2}^{1}$ and $W(0) W(\pi) \neq 0$. Then $\frac{d}{d \theta}\left(T(\theta) h_{m}^{ \pm}(\theta)\right) \in \mathcal{A}$ with

$$
\left\|\frac{d}{d \theta}\left(T(\theta) h_{m}^{ \pm}(\theta)\right)\right\|_{\mathcal{A}} \leq C(1+|m|), \quad m \in \mathbb{Z} .
$$

Proof. Since $T^{\prime}(\theta)$ and $\frac{d}{d \theta} h_{m}^{ \pm}(\theta)$ are elements of $\mathcal{A}$ for $q \in \ell_{2}^{1}$, then for $m \in \mathbb{Z}_{ \pm}$ the statement of the lemma is evident in view of (6.5). To get it for $m \in \mathbb{Z}_{\mp}$ we use (6.4), (6.5), and formula

$$
\begin{aligned}
& \frac{d}{d \theta}\left(T(\theta) h_{m}^{ \pm}(\theta)\right) \\
& \quad=\frac{d}{d \theta}\left(R^{\mp}(\theta) h_{m}^{\mp}(\theta)\right) \mathrm{e}^{ \pm 2 \mathrm{i} m \theta} \pm 2 \mathrm{i} m \mathrm{e}^{ \pm 2 \mathrm{i} m \theta} R^{\mp}(\theta) h_{m}^{\mp}(\theta)+\frac{d}{d \theta} h_{m}^{\mp}(-\theta) .
\end{aligned}
$$

The bound (6.2) for the third summand in (6.6) now follows combining Theorem 4.1, Lemmas 5.1, 6.2, and 6.3.

Theorem 6.4. Let $q \in \ell_{2}^{1}$. Then in the non-resonant case the asymptotics (1.8) hold, i.e.,

$$
\left\|\mathrm{e}^{-\mathrm{i} t H} P_{c}\right\|_{\ell_{\sigma}^{2} \rightarrow \ell_{-\sigma}^{2}}=\mathcal{O}\left(t^{-3 / 2}\right), \quad t \rightarrow \infty, \sigma>3 / 2 .
$$

Proof. We will derive (6.14) for $\left[\mathrm{e}^{-\mathrm{i} t H} P_{c}\right]^{+}$, defined in (6.6). For $\left[\mathrm{e}^{-\mathrm{i} t H} P_{c}\right]^{-}$the proof is similar. Abbreviate

$$
Z_{n, k}(\theta)=|T(\theta)|^{2} h_{k}^{+}(\theta) h_{n}^{+}(-\theta)
$$

Due to (6.6) it suffices to consider the operators $\mathcal{M}_{j}(t), j=1,2,3$, with the kernels

$$
\left[\mathcal{M}_{j}(t)\right]_{n, k}=\int_{-\pi}^{\pi} \mathrm{e}^{-\mathrm{i} t \phi_{v}(\theta)} Z_{n, k}^{j}(\theta) d \theta, \quad \phi_{v}(\theta)=2-2 \cos \theta+v \theta, v=|k-n| / t,
$$

where

$$
\begin{aligned}
& Z_{n, k}^{1}(\theta)=\frac{k-n}{\sin \theta} Z_{n, k}(\theta), \\
& Z_{n, k}^{2}(\theta)=\frac{\cos \theta}{\sin ^{2} \theta} Z_{n, k}(\theta), \\
& Z_{n, k}^{3}(\theta)=\frac{\frac{d}{d \theta} Z_{n, k}(\theta)}{\sin \theta}
\end{aligned}
$$


and obtain the bound

$$
\sum_{n, k \in \mathbb{Z}}\left[\mathcal{M}_{j}(t)\right]_{n, k}^{2} \frac{1}{(1+|n|)^{2 \sigma}(1+|k|)^{2 \sigma}} \leq C t^{-1}
$$

for any $\sigma>3 / 2$ and sufficiently large $t \geq 1$. As in the proof of Theorem 5.3, step II, we consider the integrals over

$$
J:=\{\theta:|\theta \pm \pi / 2| \leq \pi / 6\}
$$

and over $[-\pi, \pi] \backslash J$.

For the integrals over $[-\pi, \pi] \backslash J$ we apply Lemma 5.1 with $s=2$ together with the fact that $\left\|Z_{n, k}^{j}\right\|_{\mathcal{A}} \leq C(1+|n|)(1+|k|), j=1,2,3$ and obtain

$$
\left|\int_{[-\pi, \pi] \backslash J} \mathrm{e}^{-\mathrm{i} t \phi_{v}(\theta)} Z_{n, k}^{j}(\theta) d \theta\right| \leq C t^{-1 / 2}(1+|n|)(1+|k|), \quad t \geq 1 .
$$

Then the bound (6.17) for

$$
\left[\tilde{\mathcal{M}}_{j}(t)\right]_{n, k}=\int_{[-\pi, \pi] \backslash J} \mathrm{e}^{-\mathrm{i} t \phi_{v}(\theta)} Z_{n, k}^{j}(\theta) d \theta
$$

follows. To estimate the integrals over $J$ we consider the three possibilities (a) $n \leq k \leq 0$, (b) $0 \leq n \leq k$ and (c) $n \leq 0 \leq k$.

Consider the case (b). Lemma 3.1 (ii) for $s=2$ imply

$$
\left|\frac{d^{2}}{d \theta^{2}} T(\theta)\right|, \quad\left|\frac{d^{2}}{d \theta^{2}} R^{ \pm}(\theta)\right| \leq C, \quad \theta \in J .
$$

Respectively,

$$
\left|\frac{\partial}{\partial \theta} Z_{n, k}^{j}(\theta)\right| \leq C(1+k), \quad \theta \in J, 0 \leq n \leq k
$$

Then we obtain (6.17) by the same arguments as for the proof of Theorem 5.3, Step II.

Consider the case (c). Using the scattering relations (4.6) and equality (5.16) we obtain

$$
\begin{aligned}
{\left[\mathcal{M}_{j}^{ \pm}(t)\right]_{n, k}: } & =\int_{J} \mathrm{e}^{-\mathrm{i} t \phi_{v}(\theta)} Z_{n, k}^{j}(\theta) d \theta \\
& =\int_{J} \mathrm{e}^{-\mathrm{i} t \phi_{v}(\theta)} Z_{n, k, 1}^{j}(\theta) d \theta+\int_{J} \mathrm{e}^{-\mathrm{i} t \tilde{\phi}_{v}(\theta)} Z_{n, k, 2}^{j}(\theta) d \theta
\end{aligned}
$$


where $\tilde{\phi}_{v}(\theta)$ is defined in (5.15), and

$$
\begin{aligned}
& Z_{n, k, j}^{1}(\theta)=\frac{k-n}{\sin \theta} Z_{n, k, j}(\theta), \quad Z_{n, k, j}^{2}(\theta)=\frac{\cos \theta}{\sin ^{2} \theta} Z_{n, k, j}(\theta), \\
& Z_{n, k, 1}^{3}(\theta)=\frac{\frac{d}{d \theta} Z_{n, k, 1}(\theta)}{\sin \theta}, \quad Z_{n, k, 2}^{3}(\theta)=\frac{\frac{d}{d \theta} Z_{n, k, 2}(\theta)-2 \mathrm{in} Z_{n, k, 2}}{\sin \theta}
\end{aligned}
$$

with

$$
\begin{aligned}
& Z_{n, k, 1}(\theta)=T(\theta) h_{k}^{+}(\theta) h_{n}^{-}(\theta), \\
& Z_{n, k, 2}(\theta)=T(\theta) R^{-}(-\theta) h_{k}^{+}(\theta) h_{n}^{-}(-\theta) .
\end{aligned}
$$

Lemma 3.1 (ii) and (6.18) imply

$$
\left|\frac{\partial}{\partial \theta} Z_{n, k, 1}^{j}(\theta)\right|,\left|\frac{\partial}{\partial \theta} Z_{n, k, 2}^{j}(\theta)\right| \leq C(1+\max \{|n|,|k|\}), \quad \theta \in J .
$$

Hence (6.17) for the case (c) also follows.

It remains to consider the case (a). Denote

$$
\hat{\phi}_{v}(\theta)=2-2 \cos \theta+\hat{v} \theta, \text { where } \hat{v}(\theta)=-|k-n| / t \leq 0 .
$$

The scattering relations (4.6) now imply

$$
\begin{aligned}
{\left[\mathcal{M}_{j}^{ \pm}(t)\right]_{n, k}:=} & \int_{J} \mathrm{e}^{-\mathrm{i} t \phi_{v}(\theta)} Z_{n, k}^{j}(\theta) d \theta \\
= & \int_{J} \mathrm{e}^{-\mathrm{i} t \phi_{v}(\theta)} X_{n, k, 1}^{j}(\theta) d \theta \\
& +\int_{J} \mathrm{e}^{-\mathrm{i} t \tilde{\phi}_{v}(\theta)} X_{n, k, 2}^{j}(\theta) d \theta \\
& +\int_{J} \mathrm{e}^{-\mathrm{i} t \hat{\phi}_{v}(\theta)} X_{n, k, 3}^{j}(\theta) d \theta,
\end{aligned}
$$

where

$$
\begin{aligned}
& X_{n, k, j}^{1}(\theta)=\frac{k-n}{\sin \theta} X_{n, k, j}(\theta), \\
& X_{n, k, j}^{2}(\theta)=\cos \theta \frac{X_{n, k, j}(\theta}{\sin ^{2} \theta}, \\
& X_{n, k, 1}^{3}(\theta)=\frac{\frac{d}{d \theta} X_{n, k, 1}(\theta)}{\sin \theta}, \\
& X_{n, k, 3}^{3}(\theta)=\frac{\frac{d}{d \theta} X_{n, k, 3}(\theta)+2 \mathrm{i}(k-n) X_{n, k, 3}}{\sin \theta} \\
& X_{n, k, 2}^{3}(\theta)=\frac{\frac{d}{d \theta} X_{n, k, 2}(\theta)+2 \mathrm{i} k R^{-}(\theta) h_{k}^{-}(\theta) h_{n}^{-}(\theta)-2 \mathrm{i} n R^{-}(-\theta) h_{k}^{-}(-\theta) h_{n}^{-}(-\theta)}{\sin \theta}
\end{aligned}
$$


and

$$
\begin{aligned}
& X_{n, k, 1}(\theta)=h_{k}^{-}(-\theta) h_{n}^{-}(\theta), \\
& X_{n, k, 2}(\theta)=R^{-}(-\theta) h_{k}^{-}(-\theta) h_{n}^{-}(-\theta)+R^{-}(\theta) h_{k}^{-}(\theta) h_{n}^{-}(\theta), \\
& X_{n, k, 3}(\theta)=\left|R^{-}(\theta)\right|^{2} h_{k}^{-}(\theta) h_{n}^{-}(-\theta) .
\end{aligned}
$$

From Lemma 3.1 (ii) and (6.18) it follows that

$$
\left|\frac{\partial}{\partial \theta} X_{n, k, m}^{j}(\theta)\right| \leq C(1+|n|)(1+|k|), \quad \theta \in J .
$$

The integrals with the phase functions $\phi_{v}(\theta)$ and $\tilde{\phi}_{v}(\theta)$ can been estimated similarly as in the previous cases. Since $\hat{v} \leq 0$, then in order to estimate the integrals with the phase functions $\hat{\phi}_{v}(\theta)$ we can interchange the methods for $|\theta-\pi / 2| \leq$ $\pi / 6$ and for $|\theta+\pi / 2| \leq \pi / 6$.

\section{Wave equation}

Here we extend our main results to the wave equation (1.2).

7.1. Free wave equation. Set

$$
\mathbf{u}_{n}(t)=\left(u_{n}(t), \dot{u}_{n}(t)\right) .
$$

Then (1.2) with $q=0$ reads

$$
\mathrm{i} \dot{\mathbf{u}}(t)=\mathbf{H}_{0} \mathbf{u}(t), \quad t \in \mathbb{R},
$$

where

$$
\mathbf{H}_{0}=\left(\begin{array}{cc}
0 & \mathrm{i} \\
\mathrm{i}\left(\Delta_{L}-\mu^{2}\right) & 0
\end{array}\right) .
$$

The continuous spectrum of $\mathbf{H}_{0}$ coincides with $\bar{\Gamma}$, where

$$
\Gamma=\left(-\sqrt{\mu^{2}+4},-\mu\right) \cup\left(\mu, \sqrt{\mu^{2}+4}\right) .
$$

The resolvent

$$
\mathbf{R}_{0}(\omega)=\left(\mathbf{H}_{0}-\omega\right)^{-1}
$$

can be expressed in terms of $\mathcal{R}_{0}(\omega)=\left(H_{0}-\omega\right)^{-1}$, see [13],

$$
\mathbf{R}_{0}(\omega)=\left(\begin{array}{cc}
\omega \mathcal{R}_{0}\left(\omega^{2}-\mu^{2}\right) & \mathrm{i} \mathcal{R}_{0}\left(\omega^{2}-\mu^{2}\right) \\
-\mathrm{i}\left(1+\omega^{2} \mathcal{R}_{0}\left(\omega^{2}-\mu^{2}\right)\right) & \omega \mathcal{R}_{0}\left(\omega^{2}-\mu^{2}\right)
\end{array}\right) .
$$


Note that factorizing $H_{0}$ according to $H_{0}-\mu^{2}=A^{*} A$ (cf. [20, Sect. 11.1]) we can use $\left(u_{n}(t), A \dot{u}_{n}(t)\right)$ to write (7.1) in self-adjoint form. We refer to [6] and the references therein for further details.

Denote by $\mathbf{l}_{\sigma}^{p}=\ell_{\sigma}^{p} \oplus \ell_{\sigma}^{p}, p \geq 1, \sigma \in \mathbb{R}$.

Lemma 7.1. Let $\mu>0$. Then the following asymptotics hold

$$
\begin{aligned}
&\left\|\mathrm{e}^{-\mathrm{i} t \mathbf{H}_{0}}\right\|_{\mathbf{l}^{1} \rightarrow \mathbf{l}^{\infty}}=\mathcal{O}\left(t^{-1 / 3}\right), \quad t \rightarrow \infty, \\
&\left\|\mathrm{e}^{-\mathrm{i} t \mathbf{H}_{0}}\right\|_{\mathbf{l}_{\sigma}^{2} \rightarrow \mathbf{l}_{-\sigma}^{2}}=\mathcal{O}\left(t^{-1 / 2}\right), \quad t \rightarrow \infty, \sigma>1 / 2 .
\end{aligned}
$$

Proof. As in the proof of Proposition 2.1 we consider $t \geq 1$ and apply the spectral representation:

$$
\mathrm{e}^{-\mathrm{i} t \mathbf{H}_{0}}=\frac{1}{2 \pi \mathrm{i}} \int_{\Gamma} \mathrm{e}^{-\mathrm{i} t \omega}\left(\mathbf{R}_{\mathbf{0}}(\omega+\mathrm{i} 0)-\mathbf{R}_{0}(\omega-\mathrm{i} 0)\right) d \omega .
$$

We prove asymptotics (7.3) and (7.4) only for the entry $\left[\mathrm{e}^{-\mathrm{i} t \mathbf{H}_{0}}\right]^{12}$. The other entries of the matrix $\mathrm{e}^{-\mathrm{i} t \mathbf{H}_{0}}$ can be treated similarly.

Let $\theta_{+}=\theta_{+}\left(\omega^{2}-\mu^{2}\right) \in[-\pi, 0]$ be the solution of $2-2 \cos \theta=\omega^{2}-\mu^{2}$ and $\theta_{-}=-\theta_{+}$. Due to (2.1) we have

$$
\left[\mathrm{e}^{-\mathrm{i} t \mathbf{H}_{0}}\right]_{n, k}^{12}=\frac{1}{2 \pi} \int_{\Gamma} \mathrm{e}^{-\mathrm{i} t \omega}\left(\frac{\mathrm{e}^{-\mathrm{i} \theta_{+}|n-k|}}{\sin \theta_{+}}-\frac{\mathrm{e}^{-\mathrm{i} \theta_{-}|n-k|}}{\sin \theta_{-}}\right) d \omega=I_{-}+I_{+},
$$

where

$$
I_{ \pm}:=-\frac{1}{2 \pi} \int_{-\pi}^{\pi} \frac{\mathrm{e}^{ \pm \mathrm{i} t g(\theta)-\mathrm{i} \theta|n-k|} d \theta}{g(\theta)}, \quad g(\theta):=\sqrt{2-2 \cos \theta+\mu^{2}}
$$

S TEP 1. To prove (7.3) it suffices to obtain the bound

$$
\sup _{n, k}\left|\left[\mathrm{e}^{-\mathrm{i} t \mathbf{H}_{0}}\right]_{n, k}^{12}\right| \leq C t^{-1 / 3}, \quad t \geq 1
$$

We consider only the integral $I_{-}$. Abbreviate

$$
v:=\frac{|n-k|}{t} \geq 0
$$


and set

$$
\varkappa=\left(2+\mu^{2}-\sqrt{4 \mu^{2}+\mu^{4}}\right) / 2,
$$

$0<\varkappa<1$. It is easy to check that if

$$
v \neq v_{0}:=\sqrt{x}
$$

then the phase function

$$
\Phi_{v}(\theta)=g(\theta)+v \theta
$$

where $g(\theta)$ is defined in (7.6), has at most two non-degenerate stationary points. In the case $v=v_{0}$ there exists a unique degenerate stationary point $\theta_{0}=-\arccos \varkappa$, $-\pi / 2<\theta_{0}<0$, such that $\Phi^{\prime \prime \prime}\left(\theta_{0}\right)=\sqrt{\varkappa} \neq 0$. Moreover, $g(\theta)>\mu>0$, and therefore $g^{-1}(\theta)$ is a smooth function. Hence (7.7) follows from the van der Corput lemma.

STEP 2. To prove (7.4) we divide the domain of integration in $I_{-}$into the domains $J=\left\{\theta:\left|\theta-\theta_{0}\right| \leq v\left|\theta_{0}\right|\right\}$ and $[-\pi, \pi] \backslash J$, where $v=v(\mu), 0<v \leq 1$, will be specified below. We further divide the domain $J$ into subdomains $t_{j} \leq\left|\theta-\theta_{0}\right| \leq$ $t_{j+1}, 0 \leq j \leq N$, where $t_{j}$ for $j=1, \ldots, N$ is chosen as in Lemma 2.2, and $t_{N+1}=v\left|\theta_{0}\right|$. The asymptotics (7.4) for the part over $[-\pi, \pi] \backslash J$ follow from the stationary phase method. To get (7.4) for

$$
\left[\mathbf{K}_{j}(t)\right]_{n, k}=\int_{t_{j} \leq\left|\theta-\theta_{0}\right| \leq t_{j+1}} \mathrm{e}^{-\mathrm{i} t \Phi_{v}(\theta)} \frac{d \theta}{g(\theta)}, \quad 1 \leq j \leq N,
$$

we consider $\left|v_{0}-v\right| \leq \frac{1}{2} v_{0} t_{j} t^{\varepsilon}$ and $\left|v_{0}-v\right| \geq \frac{1}{2} v_{0} t_{j} t^{\varepsilon}$ separately. The first case is identical to the first case of Lemma 2.2. In the second case we apply integration by parts similarly to (2.17). Namely, we have to estimate: (a) $\left|\Phi_{v}^{\prime}(\theta)\right|^{-1}$ at the points $\theta_{0} \pm t_{j}$ and $\theta_{0} \pm t_{j+1}$, and (b) the integral of the function $\left|\Phi_{v}^{\prime \prime}(\theta)\right|\left(\Phi_{v}^{\prime}(\theta)\right)^{-2}$ between these points. But since the function $\Phi_{v}^{\prime \prime}(\theta)$ does not change its sign on the intervals $\left[\theta_{0}+t_{j}, \theta_{0}+t_{j+1}\right]$ and $\left[\theta_{0}-t_{j+1}, \theta_{0}-t_{j}\right]$, then the antiderivative of the function $\left|\Phi_{v}^{\prime \prime}(\theta)\right|\left(\Phi_{v}^{\prime}(\theta)\right)^{-2}$ is equal up to a sign to the function $\left(\Phi_{v}^{\prime}(\theta)\right)^{-1}$. Thus, it is sufficient to consider the case (a) only.

We have $\Phi_{v}(\theta)=g(\theta)+v \theta$, therefore

$$
\begin{aligned}
\Phi_{v}^{\prime}(\theta) & =g^{\prime}(\theta)+v \\
& =g^{\prime}\left(\theta_{0}\right)+\frac{1}{2} g^{\prime \prime \prime}(\tilde{\theta})\left(\theta-\theta_{0}\right)^{2}+v \\
& =\frac{1}{2} g^{\prime \prime \prime}(\tilde{\theta})\left(\theta-\theta_{0}\right)^{2}+v-v_{0} .
\end{aligned}
$$


Here we used formulas $g^{\prime}\left(\theta_{0}\right)=-v_{0}$ and $g^{\prime \prime}\left(\theta_{0}\right)=0$. Hence for large $t$

$$
\begin{aligned}
\left|\Phi_{v}^{\prime}\left(\theta_{0} \pm t_{j+s}\right)\right| & \geq\left|v-v_{0}\right|-C t_{j+s}^{2} \\
& \geq t_{j}\left(\frac{1}{2} v_{0} t^{\varepsilon}-C\right) \\
& \geq C_{1} t_{j}, \quad j=1, \ldots, N-1, s=0,1,
\end{aligned}
$$

and then

$$
\left|\left[\mathbf{K}_{j}(t)\right]_{n, k}\right| \leq C t^{-1} t_{j}^{-1} \leq C t^{-1 / 2}, \quad j=1, \ldots, N-1
$$

as in (2.19).

In the case $j=N$ we have $\left|v-v_{0}\right| \geq \frac{1}{2} v_{0}$. Further,

$$
\Phi_{v}^{\prime}\left(\theta_{0} \pm t_{N+1}\right)=\frac{1}{2} g^{\prime \prime \prime}(\tilde{\theta})\left(v\left|\theta_{0}\right|\right)^{2}+v-v_{0}
$$

Since $\left|g^{\prime \prime \prime}(\theta)\right| \leq G=G(\mu), \theta \in[-\pi, \pi]$, then we can choose

$$
v=\min \left\{1, \sqrt{\frac{2 v_{0}}{3 G \theta_{0}^{2}}}\right\}
$$

to obtain $\left|\Phi_{v}^{\prime}\left(\theta_{0} \pm t_{N+1}\right)\right| \geq \frac{1}{6} v_{0}$. Respectively, $\left|\Phi_{v}^{\prime}\left(\theta_{0} \pm t_{N+1}\right)\right|^{-1} \leq 6 / v_{0}$, and hence

$$
\left|\left[\mathbf{K}_{N}(t)\right]_{n, k}\right| \leq C t^{-1}
$$

Remark 7.2. The solution of the free wave equation (7.1), corresponding to $\mu=$ 0 , does not decay as $t \rightarrow \pm \infty$. In fact, the first component of the solution is given by

$$
u_{n}(t)=\sum_{m \in \mathbb{Z}} c_{n-m}(t) u_{m}(0)+s_{n-m}(t) \dot{u}_{m}(0)
$$

where

$$
\begin{aligned}
c_{n}(t) & =\frac{1}{2 \pi} \int_{-\pi}^{\pi} \cos (\sqrt{1-\cos \theta} \sqrt{2} t) \mathrm{e}^{\mathrm{i} \theta n} d \theta=J_{2|n|}(2 t), \\
s_{n}(t) & =\frac{1}{2 \pi} \int_{-\pi}^{\pi} \frac{\sin (\sqrt{1-\cos \theta} \sqrt{2} t)}{\sqrt{1-\cos \theta}} \mathrm{e}^{\mathrm{i} \theta n} d \theta=\int_{0}^{t} c_{n}(s) d s \\
& =\frac{t^{2|n|+1}}{2^{|n|}(|n|+1) !}{ }_{1} F_{2}\left(\frac{2|n|+1}{2} ;\left(\frac{2|n|+3}{2}, 2|n|+1\right) ;-t^{2}\right) .
\end{aligned}
$$

Here $J_{n}(x),{ }_{p} F_{q}(\underline{u} ; \underline{v} ; x)$ denote the Bessel and generalized hypergeometric functions, respectively. In particular, while $c_{n}(t)=O\left(t^{-1 / 2}\right)$ for fixed $n$, we have $s_{n}(t)=\frac{1}{2}+O\left(t^{-1 / 2}\right)$ for fixed $n$. 
7.2. Perturbed wave equation. In matrix form (1.2) reads

$$
\mathrm{i} \dot{\mathbf{u}}(t)=\mathbf{H u}(t), \quad t \in \mathbb{R},
$$

where

$$
\mathbf{H}=\left(\begin{array}{cc}
0 & \mathrm{i} \\
\mathrm{i}\left(\Delta_{L}-\mu^{2}-q\right) & 0
\end{array}\right) .
$$

The resolvent $\mathbf{R}(\omega)=(\mathbf{H}-\omega)^{-1}$ can be expressed in terms of $\mathcal{R}(\omega)=(H-\omega)^{-1}$ (see [13]):

$$
\mathbf{R}(\omega)=\left(\begin{array}{cc}
\omega \mathcal{R}\left(\omega^{2}-\mu^{2}\right) & \mathrm{i} \mathcal{R}\left(\omega^{2}-\mu^{2}\right) \\
-\mathrm{i}\left(1+\omega^{2} \mathcal{R}\left(\omega^{2}-\mu^{2}\right)\right) & \omega \mathcal{R}\left(\omega^{2}-\mu^{2}\right)
\end{array}\right) .
$$

Representation (7.13) and Lemma 3.3 imply the limiting absorption principle for the perturbed resolvent:

Lemma 7.3. Suppose $q \in \ell^{1}$. Then for $\omega \in \Gamma$ the convergence

$$
\mathbf{R}(\omega \pm \mathrm{i} \varepsilon) \rightarrow \mathbf{R}(\omega \pm \mathrm{i} 0), \quad \varepsilon \rightarrow 0+,
$$

holds in $\mathcal{L}\left(\mathbf{l}_{\sigma}^{2}, \mathbf{l}_{-\sigma}^{2}\right)$ with $\sigma>1 / 2$.

For the dynamical group associated with the perturbed wave equation (7.12) the spectral representation of type (5.5) holds:

$$
\mathrm{e}^{-\mathrm{i} t \mathbf{H}} \mathbf{P}_{c}=\frac{1}{2 \pi \mathrm{i}} \int_{\Gamma} \mathrm{e}^{-\mathrm{i} t \omega}(\mathbf{R}(\omega+\mathrm{i} 0)-\mathbf{R}(\omega-\mathrm{i} 0)) d \omega .
$$

Here $\mathbf{P}_{c}$ is the projection onto the continuous spectrum of $\mathbf{H}^{2}$. Next, we prove asymptotics of type (1.3) and (1.8) for (7.12).

Theorem 7.4. Let $\mu>0$ and $q \in \ell_{1}^{1}$. Then the following asymptotics holds

$$
\left\|\mathrm{e}^{-\mathrm{i} t \mathbf{H}} \mathbf{P}_{c}\right\|_{\mathbf{1}^{1} \rightarrow \mathbf{1}^{\infty}}=\mathcal{O}\left(t^{-1 / 3}\right), \quad t \rightarrow \infty .
$$

and

$$
\left\|\mathrm{e}^{-\mathrm{i} t \mathbf{H}} \mathbf{P}_{c}\right\|_{\mathbf{l}_{\sigma}^{2} \rightarrow \mathbf{l}_{-\sigma}^{2}}=\mathcal{O}\left(t^{-1 / 2}\right), \quad t \rightarrow \infty, \quad \sigma>1 / 2 .
$$


Proof. STEP I. Due to the representation (7.14) and formula (3.11) it suffices to obtain (7.15)-(7.16) for the operator with the kernel

$$
\begin{aligned}
{[\mathbf{K}(t)]_{n, k} } & =\int_{\mu}^{\sqrt{\mu^{2}+4}} \mathrm{e}^{-\mathrm{i} t \omega}\left[\frac{f_{k}^{+}\left(\theta_{+}\right) f_{n}^{-}\left(\theta_{+}\right)}{W\left(\theta_{+}\right)}-\frac{f_{k}^{+}\left(\theta_{-}\right) f_{n}^{-}\left(\theta_{-}\right)}{W\left(\theta_{-}\right)}\right] d \omega \\
& =-\int_{-\pi}^{\pi} \mathrm{e}^{-\mathrm{i} t g(\theta)} \frac{f_{k}^{+}(\theta) f_{n}^{-}(\theta)}{W(\theta)} \frac{\sin \theta d \theta}{g(\theta)} \\
& =\frac{\mathrm{i}}{2} \int_{-\pi}^{\pi} \mathrm{e}^{-\mathrm{i} t \Phi_{v}(\theta)} h_{k}^{+}(\theta) h_{n}^{-}(\theta) T(\theta) \frac{d \theta}{g(\theta)}, \quad n \leq k,
\end{aligned}
$$

where the phase function $\Phi_{v}(\theta)$ is defined in (7.8). For (7.15) we need to prove that

$$
\sup _{n \leq k}\left|[\mathbf{K}(t)]_{n, k}\right| \leq C t^{-1 / 3}, \quad t \geq 1 .
$$

Just as in the proof of Theorem 5.3 (i) we consider three different cases (a), (b) and (c). Using the properties of the phase function obtained in Lemma 7.1 one can now proceed as in the proof of Theorem 5.3 (i).

SteP II. Recall that $-\pi / 2<\theta_{0}<0$. Denote $\mathbf{J}:=\left\{\theta:\left|\theta \pm \theta_{0}\right| \leq v\left|\theta_{0}\right|\right\}$, where $v=v(\mu)$ is defined in Lemma 7.1. We represent $[\mathbf{K}(t)]_{n, k}$ as the sum

$$
[\mathbf{K}(t)]_{n, k}=\left[\mathbf{K}^{ \pm}(t)\right]_{n, k}+[\tilde{\mathbf{K}}(t)]_{n, k}
$$

where

$$
\begin{aligned}
{\left[\mathbf{K}^{ \pm}(t)\right]_{n, k} } & =\frac{1}{2 \pi} \int_{\left|\theta \pm \theta_{0}\right| \leq \delta} \mathrm{e}^{-\mathrm{i} t \Phi_{v}(\theta)} Y_{n, k}(\theta) \frac{d \theta}{g(\theta)}, \\
{[\tilde{\mathbf{K}}(t)]_{n, k} } & =\frac{1}{2 \pi} \int_{\theta \in[-\pi, \pi] \backslash \mathbf{J}} \mathrm{e}^{-\mathrm{i} t \Phi_{v}(\theta)} Y_{n, k}(\theta) \frac{d \theta}{g(\theta)},
\end{aligned}
$$

and $Y_{n, k}(\theta)=h_{k}^{+}(\theta) h_{n}^{-}(\theta) T(\theta)$ as above. Note that the bound (5.13) holds for $\theta \in \mathbf{J}$ also. Hence one can now proceed as in the proof of Theorem 5.3 (ii) to obtain (7.15).

Remark 7.5. In the case $\mu=0$ the factor $\sin (\theta / 2)$ in the denominator of (7.17) implies that we cannot get (7.15) and (7.16) for $\left[\mathrm{e}^{-\mathrm{i} t \mathbf{H}} \mathbf{P}_{c}\right]^{12}$ in this case. Nevertheless, the analogous expression for the other entries of $\left[\mathrm{e}^{-\mathrm{i} t \mathbf{H}} \mathbf{P}\right]$ does not contain this factor in the denominator and hence asymptotics (7.15) and (7.16) with the decay rate $t^{-1 / 3}$ hold for these entries. 
Now we consider the non-resonant case and obtain asymptotics of type (1.9) and (1.10) for equation (7.12). To this end note that $\mathbf{H}$ has a resonance at a boundary point of the continuous spectrum $\bar{\Gamma}$ if and only if $H$ has a resonance at the corresponding boundary point of its continuous spectrum $[0,4]$.

Theorem 7.6. $i)$ Let $\mu \geq 0, q \in \ell_{2}^{1}$. Then in the non-resonant case the following asymptotics hold

$$
\left\|\mathrm{e}^{-\mathrm{i} t \mathbf{H}} \mathbf{P}_{c}\right\|_{\mathbf{1}_{1}^{1} \rightarrow \mathbf{1}_{-1}^{\infty}}=\mathcal{O}\left(t^{-4 / 3}\right), \quad t \rightarrow \infty .
$$

ii) Let $\mu>0$ and $q \in \ell_{2}^{1}$. Then in the non-resonant case and for any $\sigma>3 / 2$ the following asymptotics hold

$$
\left\|\mathrm{e}^{-\mathrm{i} t \mathbf{H}} \mathbf{P}_{c}\right\|_{\mathbf{l}_{\sigma}^{2} \rightarrow \mathbf{l}_{-\sigma}^{2}}=\mathcal{O}\left(t^{-3 / 2}\right), \quad t \rightarrow \infty .
$$

iii) In the case $\mu=0$ the asymptotics (7.20) hold under the stronger conditions $q \in \ell_{3}^{1}$ and $\sigma>5 / 2$.

Proof. We consider $\left[\mathrm{e}^{-\mathrm{i} t \mathbf{H}} \mathbf{P}_{c}\right]^{12}$ and the case $n \leq k$ only. As in the proof of Theorems 6.1 and 6.4 we need to consider the operators $\mathbf{M}_{j}(t)$ with the kernels

$$
\left[\mathbf{M}_{j}(t)\right]_{n, k}=t^{-1} \int_{-\pi}^{\pi} \mathrm{e}^{-\mathrm{i} t \Phi_{v}(\theta)} Z_{n, k}^{j}(\theta) d \theta,
$$

where $Z_{n, k}$ and $\Phi_{v}(\theta)$ are defined in (6.15)-(6.16) and (7.8), and obtain the asymptotics

$$
\begin{gathered}
\left\|\mathbf{M}_{j}(t)\right\|_{\mathbf{l}_{1}^{1} \rightarrow \mathbf{l}_{-1}^{\infty}}=\mathcal{O}\left(t^{-4 / 3}\right), \quad t \rightarrow \infty, \\
\left\|\mathbf{M}_{j}(t)\right\|_{\mathbf{l}_{\sigma}^{2} \rightarrow \mathbf{l}_{-\sigma}^{2}}=\mathcal{O}\left(t^{-3 / 2}\right), \quad t \rightarrow \infty, \sigma>3 / 2 .
\end{gathered}
$$

The asymptotics (7.21) for $\mu \geq 0$ and the asymptotics (7.22) in the case $\mu>0$ can be established as in the proofs of Theorems 6.1 and 6.4.

In the case $\mu=0$ we have $\theta_{0}=0$ and $\mathbf{J}=\mathbf{J}_{0}=\{\theta:|\theta| \leq \pi / 6\}$. The integrals over $[-\pi, \pi] \backslash \mathbf{J}_{0}$ can be estimated as in the case $\mu>0$. Concerning the integral over $\mathbf{J}_{0}$ we will not split it as in (6.6) but consider the whole integral

$$
[\mathbf{M}(t)]_{n, k}=t^{-1} \int_{\mathbf{J}_{0}} \mathrm{e}^{-2 \mathrm{i} t \sin (\theta / 2)} \frac{d}{d \theta}\left(\mathrm{e}^{-\mathrm{i} \theta(k-n)} \frac{|T(\theta)|^{2}}{\sin \theta} h_{k}^{+}(\theta) h_{n}^{+}(-\theta)\right) d \theta .
$$

We apply integration by parts once more and obtain the asymptotics of type (7.22) for $\mathbf{M}(t)$ with decay rate $t^{-2}$ and with $\sigma>5 / 2$ if we prove that

$$
\left|\frac{d^{2}}{d \theta^{2}}\left(\frac{|T(\theta)|^{2}}{\sin ^{2} \theta} h_{k}^{+}(\theta) h_{n}^{+}(-\theta) \sin \theta\right)\right| \leq C\left(1+|n|^{2}\right)\left(1+|k|^{2}\right), \quad \theta \in \mathbf{J}_{0} .
$$


Since $T(\theta) / \sin (\theta)=2 \mathrm{i} / W(\theta)$ and its first and second derivatives are bounded for $q \in \ell_{3}^{1}$, it suffices to prove that

$$
\left|\frac{d^{2}}{d \theta^{2}}\left(h_{k}^{+}(\theta) h_{n}^{+}(-\theta) \sin \theta\right)\right| \leq C\left(1+|n|^{2}\right)\left(1+|k|^{2}\right), \quad \theta \in \mathbf{J}_{0},
$$

which follows from (3.3) with $p=0,1$ and from the following bound

$$
\left|\frac{d^{2}}{d \theta^{2}}\left(h_{m}^{+}(\theta) \sin \theta\right)\right| \leq C \max \left\{-m^{2}, 1\right\}, \quad \theta \in \mathbf{J}_{0} .
$$

The last bound in the case $m \geq 0$ follows from (3.3) with $p=2$. In the case $m<0$ one needs to apply the scattering relation as before.

Acknowledgments. We are very grateful to Hans Georg Feichtinger, Michael Goldstein, Wilhelm Schlag, and Andreas Seeger for helpful discussions and to Michael Goldberg for hints with respect to the literature. We are also indebted to the referee for challenging us to improve our results.

\section{References}

[1] S. Cuccagna and M. Tarulli, On asymptotic stability of standing waves of discrete Schrödinger equation in Z Z. SIAM J. Math. Anal. 41 (2009), 861-885. MR 2529950 Zbl 1189.35303

[2] P. Deift and E. Trubowitz, Inverse scattering on the line. Comm. Pure Appl. Math. 32 (1979), 121-251. MR 0512420 Zbl 0388.34005

[3] I. Egorova and L. Golinskii, On the location of the discrete spectrum for complex Jacobi matrices. Proc. Amer. Math. Soc. 133 (2005), 3635-3641. MR 2163601 Zbl 1090.47018

[4] I. Egorova, J. Michor, and G. Teschl, Scattering theory with finite-gap backgrounds: Transformation operators and characteristic properties of scattering data. Math. Phys. Anal. Geom. 16 (2013), 111-136. MR 3063954 Zbl 1259.34089

[5] I. Egorova, E. Kopylova, V. Marchenko, and G. Teschl, Dispersion estimates for one-dimensional Schrödinger and Klein-Gordon equations revisited. Preprint 2014. arXiv:1411.0021 [math.AP]

[6] F. Gesztesy, J. A. Goldstein, H. Holden, and G. Teschl, Abstract wave equations and associated Dirac-type operators. Ann. Mat. Pura Appl. (4) 191 (2012), no. 4, 631-676. MR 2993967 Zbl 1262.35174

[7] M. Goldberg, Transport in the one-dimensional Schrödinger equation. Proc. Amer. Math. Soc. 135 (2007), no. 10, 3171-3179. MR 2322747 Zbl 1123.35046 
[8] M. Goldberg and W. Schlag, Dispersive estimates for Schrödinger operators in dimensions one and three. Commun. Math. Phys. 251 (2004), 157-178. MR 2096737 Zbl 1086.81077

[9] A. Jensen and T. Kato, Spectral properties of Schrödinger operators and timedecay of the wave functions. Duke Math. J. 46 (1979), 583-611. MR 0544248 Zbl 0448.35080

[10] P. G. Kevrekidis, D. E. Pelinovsky, and A. Stefanov, Asymptotic stability of small bound states in the discrete nonlinear Schrödinger equation. SIAM J. Math. Anal. 41 (2009), no. 5, 2010-2030. MR 2578796 Zbl 1197.35270

[11] M. Keel and T. Tao, Endpoint Strichartz estimates. Amer. J. Math. 120 (1998), 955980. MR 1646048 Zbl 0922.35028

[12] A. Komech and E. Kopylova, Dispersion decay and scattering theory. John Wiley \& Sons, Hoboken, N.J., 2012. MR 3015024 Zbl 06042927

[13] A. Komech, E. Kopylova, and M. Kunze, Dispersive estimates for 1D discrete Schrödinger and Klein-Gordon equations. Appl. Anal. 85 (2006), no. 12, 1487-1508. MR 2282998 Zbl 1121.39015

[14] E. Kopylova, On the asymptotic stability of solitary waves in the discrete Schrödinger equation coupled to a nonlinear oscillator. Nonlinear Anal. 71 (2009), no. 7-8, 3031-3046. MR 2532828 Zbl 1167.35515

[15] D. Pelinovsky and A. Sakovich, Internal modes of discrete solitons near the anticontinuum limit of the dNLS equation. Phys. D 240 (2011), 265-281. MR 2748734 Zbl 1234.37051

[16] D. Pelinovsky and A. Stefanov, On the spectral theory and dispersive estimates for a discrete Schrödinger equation in one dimension. J. Math. Phys. 49 (2008), no. 11, article id. 113501, 17 pp. MR 2468536 Zbl 1159.81336

[17] W. Schlag, Dispersive estimates for Schrödinger operators: a survey. J. Bourgain, C. E. Kenig and S. Klainerman (eds.), Mathematical aspects of nonlinear dispersive equations. Papers from the CMI/IAS Workshop on Mathematical Aspects of Nonlinear PDEs held in Princeton, N.J., 2004. Annals of Mathematics Studies, 163. Princeton University Press, Princeton, N.J., 2007, 255-285. MR 2333215 MR 2332225 (collection) Zbl 1143.35001 Zbl 1113.35005 (collection)

[18] A. Stefanov and P. G. Kevrekidis, Asymptotic behaviour of small solutions for the discrete nonlinear Schrödinger and Klein-Gordon equations. Nonlinearity 18 (2005), no. 4, 1841-1857. MR 2150357 Zbl 1181.35266

[19] E. M. Stein, Harmonic analysis: real-variable methods, orthogonality, and oscillatory integrals. With the assistance of Timothy S. Murphy. Princeton Mathematical Series, 43. Monographs in Harmonic Analysis, III. Princeton University Press, Princeton, N.J., 1993. MR 1232192 Zbl 0821.42001

[20] G. Teschl, Jacobi operators and completely integrable nonlinear lattices. Mathematical Surveys and Monographs, 72. American Mathematical Society, Providence, R.I., 2000. MR 1711536 Zbl 1056.39029 
[21] G. N. Watson, A treatise on the theory of Bessel functions. Cambridge University Press, Cambridge, 1886.

Received March 30, 2014; revised August 21, 2014

Iryna Egorova, Faculty of Mathematics, University of Vienna, Oskar-Morgenstern-Platz 1, 1090 Wien, Austria

Institute for Low Temperature Physics, 47, Lenin ave, 61103 Kharkiv, Ukraine

e-mail: iraegorova@gmail.com

Elena Kopylova, Faculty of Mathematics, University of Vienna, Oskar-Morgenstern-Platz 1, 1090 Wien, Austria

Institute for Information Transmission Problems, Russian Academy of Sciences, Moscow 127994, Russia

home page: http://www.mat.univie.ac.at/ ek/

e-mail: Elena.Kopylova@univie.ac.at

Gerald Teschl, Faculty of Mathematics, University of Vienna,

Oskar-Morgenstern-Platz 1, 1090 Wien, Austria

International Erwin Schrödinger Institute for Mathematical Physics,

Boltzmanngasse 9, 1090 Wien, Austria

home page: http://www.mat.univie.ac.at/ gerald/

e-mail: Gerald.Teschl@univie.ac.at 